\title{
INDEX REDUCTION FOR BRAUER CLASSES VIA STABLE SHEAVES
}

\author{
DANIEL KRASHEN AND MAX LIEBLICH
}

\begin{abstract}
We use twisted sheaves to study the problem of index reduction for Brauer classes. In general terms, this problem may be phrased as follows: given a field $k$, a $k$-variety $X$, and a class $\alpha \in \operatorname{Br}(k)$, compute the index of the class $\alpha_{k(X)} \in \operatorname{Br}(X)$ obtained from $\alpha$ by extension of scalars to $k(X)$. We give a general method for computing index reduction which refines classical results of Schofield and van den Bergh. When $X$ is a curve of genus 1, we use Atiyah's theorem on the structure of stable vector bundles with integral slope to show that our formula simplifies dramatically, giving a complete solution to the index reduction problem in this case. Using the twisted Fourier-Mukai transform, we show that a similarly simple formula describes homogeneous index reduction on torsors under higher-dimensional abelian varieties.
\end{abstract}

\section{CONTENTS}

1. Introduction

Acknowledgments

1.1. Simple bundles on a pullback gerbe

1.2. (Semi)stable bundles

1.3. Moduli of stable sheaves

2. Index reduction via stable twisted sheaves

2.1. Twisted Riemann-Roch

2.2. A formula for index reduction

2.3. Higher-dimensional varieties

3. Examples

1

3.1. Index reduction for local fields

3.2. Counterexamples for non-constant classes

4. Index reduction on torsors under abelian varieties and homogeneous twisted bundles 12

4.1. Twisted Fourier-Mukai transforms

4.2. Moduli of homogeneous bundles

4.3. A criterion for homogeneous index reduction

A.1. Construction of the equivalence

\section{INTRODUCTION}

Let $k$ be a field and $X$ a $k$-variety. The index reduction problem asks the following question: Given an element of the Brauer group $\alpha \in \operatorname{Br}(k)$, how does one compute the index of the class $\alpha_{k(X)}$ obtained by extending scalars from $k$ to $k(X)$ ? The implicit assumption in this question is that one may already know how to compute the index of an algebra defined over $k$ (and its finite field extensions). A reasonable answer therefore would be to describe the index of $\alpha_{k(X)}$ in terms of the indicies of some list of auxiliary algebras defined over the ground field $k$, or finite extensions of $k$. Results of this sort have been obtained for certain hyperelliptic curves $X$ and $k$ a p-adic field in [24], and for $k$ arbitrary and $X$ a projective homogeneous variety for some linear algebraic group in [16]. In this paper we give a

Krashen was partially supported by an NSA Young Investigator's Grant.

Lieblich was partially supported by an NSF Postdoctoral Fellowship. 
solution in the case that $X$ is a curve of genus 1 (Corollary 2.2.3), and make some reductions on the computation of the index in the general case (Proposition 2.2.2).

Our method is based on the use of twisted sheaves. Before going into detail about how the theory is used in this particular case, it may be instructive to give a philosophical comparison between our method and the methods used in [16] in the case of homogeneous varieties.

To begin, we consider a Brauer class on a variety which has been obtained by extension of scalars from the ground field. In the above notation, this would be the class $\alpha_{X}$. Finding the index of $\alpha_{k(X)}$ corresponds to the finding the minimal dimension of a module for an Azumaya algebra representing the class $\alpha_{k(X)}$. Concretely, if $\alpha_{k(X)}$ is the class of a central simple algebra $A=M_{m}(D)$ for some $k(X)$ central division algebra $D$, then a module of minimal dimension would be of the form $D^{m}$ and we may compute the index of $A$ as $\operatorname{ind}(A)=\operatorname{deg}(D)=\operatorname{dim}_{k}\left(D^{m}\right) / \operatorname{deg}(A)$. The problem of finding a module of minimal size may be made more geometric by noticing that this module corresponds to a coherent sheaf of modules for an Azumaya algebra $A_{X}$ in the class $\alpha_{X}$. In [16] the authors then proceed by considering the $K$-theory of the category of such modules.

The main idea in our approach here is based on the desire to deal with sheaves of modules for $A_{X}$ is a more geometric way, and in particular, in a way which reflects more closely the study of sheaves of modules over $X$, allowing us to draw from the theory of vector bundles on $X$. To accomplish this, the algebra $A_{X}$ is replaced by a gerbe corresponding to its Brauer class $\alpha_{X}$, and correspondingly the sheaves of modules for $A_{X}$ are replaced by twisted sheaves for the gerbe. This allows us to realize our goal, as many useful facts about vector bundles and sheaves turn out to generalize to their twisted counterparts. In the case of genus 1 curves, Atiyah's classification of vector bundles on an elliptic curve plays an essential role in the problem of index reduction.

Attempting to generalize these results to torsors under higher-dimensional abelian varieties leads us to briefly study the twisted Fourier-Mukai transform. We deduce a criterion for homogeneous index reduction (see 4.3.1) which shows the stark difference between index reduction for torsors under abelian varieties and rational homogeneous spaces. We include an appendix by Bhargav Bhatt which uses the twisted Fourier-Mukai transform to study the period-index problem for torsors under abelian varieties.

In this paper we freely use the theory of twisted sheaves. For the basic facts concerning these objects and their relation to the index of a Brauer class, the reader should refer to [13].

\section{ACKNOWLEDGMENTS}

During the course of this work, we had helpful conversations with Bhargav Bhatt and Jean-Louis Colliot-Thélène.

1.1. Simple bundles on a pullback gerbe. In laying the groundwork for our constructions, it is not necessary to work over a field, and so we develop our basic machinery in the context of a base $S$ which is an algebraic space. If the reader prefers, they may simply consider the case $S=\operatorname{Spec}(k)$.

Let $f: X \rightarrow S$ be a proper flat morphism of finite presentation between algebraic spaces which is cohomologically flat in dimension 0 and $\mathscr{X} \rightarrow X$ a $\mu_{n}$-gerbe. We begin by introducing the stack $\underline{\mathrm{Spl}}_{\mathscr{X} / S}$ of simple $\mathscr{X}$-twisted sheaves and its corresponding coarse moduli space $\mathrm{Spl}_{\mathscr{X} / S}$. As we will see below (1.1.4), the natural map $\underline{\mathrm{Spl}}_{\mathscr{X} / S} \rightarrow \mathrm{Spl}_{\mathscr{X} / S}$ is a $\mathbf{G}_{m}$-gerbe, and thus thus the obstruction for lifting a point in the coarse moduli to an object of the stack may be interpreted as a Brauer class.

Definition 1.1.1. Given $T \rightarrow S$, a flat family of simple coherent $\mathscr{X}$-twisted sheaves parametrized by $T$ is a $T$-flat quasi-coherent $\mathscr{X}$-twisted sheaf of finite presentation $\mathscr{F}$ on $X_{T}$ such that

(1) the formation of $\left(f_{T}\right)_{*} \mathscr{E} n d(\mathscr{F})$ commutes with base change on $T$, and

(2) the natural map $\mathscr{O}_{T} \rightarrow\left(f_{T}\right)_{*} \mathscr{E} n d(\mathscr{F})$ is an isomorphism.

It is easy to see that the two conditions of the definition are satisfied if and only if for every point $t \rightarrow T$, the only automorphisms of $\mathscr{F}_{t}$ are scalar multiplications. (Thus, if $T$ is a local Artin scheme, the reader can check that the family is simple if and only if its restriction to the reduced structure is simple.) It is also clear that the collection of flat families of simple coherent $\mathscr{X}$-twisted sheaves is a stack in the fpqc topology on $S$-schemes. We will write $\underline{\mathrm{Spl}}_{\mathscr{X} / S}$ for this stack. 
Lemma 1.1.2. The inertia stack $\mathscr{I}\left(\underline{\mathrm{Spl}}_{\mathscr{X} / S}\right)$ is naturally isomorphic to $\mathbf{G}_{m, \underline{\mathrm{Spl}}_{\mathscr{X} / S}}$.

Proof. With the notation of [1.1.1, the map $\mathscr{O}_{T} \rightarrow\left(f_{T}\right)_{*} \mathscr{E} n d(\mathscr{F})$ gives rise to a map $\mathbf{G}_{m, T} \rightarrow\left(f_{T}\right)_{*} \operatorname{Aut}(\mathscr{F})$. This yields a natural map $\mathbf{G}_{m} \rightarrow \mathscr{I}\left(\underline{\mathrm{Spl}}_{\mathscr{X} / S}\right)$. The conditions of 1.1.1 then say precisely that this map is an isomorphism.

Lemma 1.1.3. Let $\mathscr{F}$ be a T-flat quasi-coherent sheaf of finite presentation on $X_{T}$. There is an open subscheme $U \subset T$ such that a map $T^{\prime} \rightarrow T$ factors through $U$ if and only if the pullback $\mathscr{F}_{T^{\prime}}$ is a flat family of simple coherent $\mathscr{X}$-twisted sheaves.

Proof. Since being simple is a fiberwise condition, it suffices to assume that $T$ is reduced and Noetherian and show that the set $U$ of points parametrizing simple fibers is open. The set is constructible: $f_{*} \mathscr{E} n d(\mathscr{F})$ is coherent and generically compatible with base change, as are the kernel and cokernel of the map $\mathscr{O}_{T} \rightarrow f_{*} \mathscr{E} n d(\mathscr{F})$. Nakayama's lemma immediately shows constructibility. The set is stable under generization: suppose $T$ is the spectrum of a discrete valuation ring whose closed point is in $U$. The formation of $f_{*} \mathscr{E} n d(\mathscr{F})$ is certainly compatible with passage to the generic fiber. On the other hand, $f_{*} \mathscr{E} n d(\mathscr{F})$ is a coherent sheaf on $T$ whose closed fiber is 1-dimensional. It follows from standard semicontinuity results (for possibly non-flat modules over a dvr) that the generic fiber is also 1-dimensional. From this it immediately follows that the scalars generate the endomorphisms on the generic fiber. Since $U$ is constructible and stable under generalization, we conclude that it is open, as desired.

Proposition 1.1.4. The stack $\underline{\mathrm{Spl}}_{\mathscr{X} / S}$ is a $\mathbf{G}_{m}$-gerbe over an algebraic space locally of finite presentation $\mathrm{Spl}_{\mathscr{X} / S} \rightarrow S$.

Proof. The methods of [15] show that the stack $\operatorname{Coh}_{\mathscr{X} / S}$ of all coherent sheaves on $\mathscr{X}$ is an Artin stack locally of finite presentation over the base. It is straightforward to check that the collection of $\mathscr{X}$ twisted coherent sheaves is an open substack. Applying[1.1.3, we see that $\underline{\mathrm{Spl}}_{\mathscr{X} / S}$ is an open substack of $\mathrm{Coh}_{\mathscr{X} / S}$, and thus it is an Artin stack locally of finite presentation over $S$.

Since the inertia stack is fppf over $\underline{\mathrm{Spl}}_{\mathscr{X} / S}$, it follows from standard methods (first described in the last paragraph of remark 2 in the appendix to [1]) that the sheafification $\operatorname{Spl}_{\mathscr{X} / S}$ of $\underline{\mathrm{Spl}}_{\mathscr{X} / S}$ is an algebraic space and that the natural map $\underline{\mathrm{Spl}}_{\mathscr{X} / S} \rightarrow \mathrm{Spl}_{\mathscr{X} / S}$ realizes $\underline{\mathrm{Spl}}_{\mathscr{X} / S}$ as a gerbe. It is then immediate that it is a $\mathbf{G}_{m}$-gerbe.

Definition 1.1.5. With the above notation, the cohomological Brauer class $\left[\underline{\mathrm{Spl}}_{\mathscr{X} / S}\right] \in \mathrm{H}^{2}\left(\mathrm{Spl}_{\mathscr{X} / S}, \mathbf{G}_{m}\right)$ will be called the universal obstruction.

When the gerbe $\mathscr{X} \rightarrow X$ is a pullback from $S$, we can be more precise about the structure of the universal obstruction and its variation with $\mathscr{S}$.

Proposition 1.1.6. Let $\mathscr{S} \rightarrow S$ be a $\boldsymbol{\mu}_{n}$-gerbe, and let $\widetilde{\mathscr{S}} \rightarrow S$ be the associated $\mathbf{G}_{m}$-gerbe.

(1) There is a natural isomorphism $\varphi_{\mathscr{S}}: \mathrm{Spl}_{X / S} \stackrel{\sim}{\rightarrow} \mathrm{Spl}_{X \times_{S} \mathscr{S}}$ over $S$.

(2) Via $\varphi$, there is an equality

$$
\left[\underline{\mathrm{Spl}}_{X \times_{S} \mathscr{S}}\right]-\left[\underline{\mathrm{Spl}}_{X / S}\right]=\left[\widetilde{\mathscr{S}}_{\mathrm{Spl}_{X / S}}\right] \in \mathrm{H}^{2}\left(\operatorname{Spl}_{X / S}, \mathbf{G}_{m}\right) .
$$

Proof. We will define a section of the sheaf $\operatorname{Isom}_{S}\left(\operatorname{Spl}_{X / S}, \operatorname{Spl}_{X \times_{S} \mathscr{S} / S}\right)$ by gluing local sections. If $\widetilde{\mathscr{S}}$ is trivial, so that there is an invertible $\mathscr{S}$-twisted sheaf $\mathscr{L}$, then $V \mapsto V \otimes \mathscr{L}$ defines a diagram

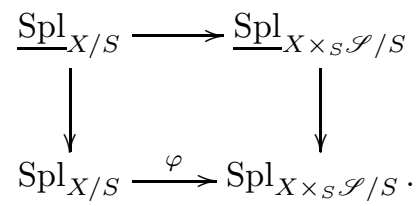

Thus, on an étale cover $U \rightarrow S$ such that $\widetilde{\mathscr{S}} \times{ }_{S} U$ is trivial, we have such a section $\varphi_{U}$. On $U \times{ }_{S} U$ we have $p_{1}^{*} \mathscr{L} \otimes p_{2}^{*} \mathscr{L}^{\vee} \cong \mathscr{M}$ with $\mathscr{M} \in \operatorname{Pic}\left(U \times_{S} U\right)$. Thus, $p_{2}^{*} \varphi_{U}^{-1} \circ p_{1}^{*} \varphi_{U}$ is the map $\operatorname{Spl}_{X / S} \rightarrow \operatorname{Spl}_{X / S}$ 
induced by twisting by $\mathscr{M}$. On the coarse moduli space, this map equals id. Thus, $\varphi_{U}$ satisfies the cocycle condition, giving rise to a global isomorphism. A similar argument shows that the resulting isomorphism is independent of the choices of $U$ and $\mathscr{L}$.

To prove the second statement, we use the results of section 2.4 of chapter IV of [10]. First, we note that the stack $\widetilde{\mathscr{S}}$ parametrizes $\mathscr{S}$-twisted invertible sheaves. (In other words, the stack $\underline{\operatorname{Hom}}_{S}^{\mathbf{G}_{m}}\left(\widetilde{\mathscr{S}}, \mathrm{BG}_{m}\right)$ of 1-morphisms of $\mathbf{G}_{m}$-gerbes is isomorphic to $\widetilde{\mathscr{S}}$.) Consider the morphism

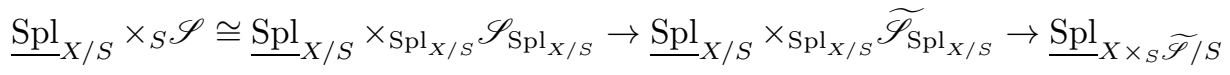

which sends a pair $(V, L)$ consisting of a flat family of simple coherent sheaves on $X$ and a flat family of invertible $\mathscr{S}$-twisted sheaves to the tensor product $V \otimes L$. This map is compatible with the product map $\mathbf{G}_{m} \times \mathbf{G}_{m} \rightarrow \mathbf{G}_{m}$ in the obvious way. In the notation of section 1.6.1 of chapter IV, the multiplication map is precisely the contracted product of the two copies of $\mathbf{G}_{m}$. By Proposition IV.2.4.1 of [10], there is an induced map of $\mathbf{G}_{m}$-gerbes

$$
\underline{\operatorname{Spl}}_{X / S} \bigwedge_{\mathrm{Spl}_{X / S}}^{C} \widetilde{\mathscr{S}}_{\mathrm{Spl}_{X / S}} \rightarrow \underline{\operatorname{Spl}}_{X \times_{S} \widetilde{\mathscr{S}} / S} .
$$

But any map of gerbes gives an equality of the associated cohomology classes. Since the contracted product gives the sum of cohomology classes, the result follows.

Corollary 1.1.7. The twisted Picard stack $\mathscr{P}_{i c_{X} \times_{S} \mathscr{S} / S}$ naturally has sheafification $\mathrm{Pic}_{X / S}$ with universal obstruction $\left[\mathscr{P}_{i c_{X / S}}\right]+\left[\widetilde{\mathscr{S}}_{\mathrm{Pic}_{X / S}}\right]$.

1.2. (Semi)stable bundles. We briefly recall the elements of the theory of stable and semistable twisted sheaves on curves. This theory is the specialization of a much more general theory of (semi)stable sheaves on arbitrary polarized gerbes (or even arbitrary polarized orbifolds), but such extreme generality will have no place in the rest of this paper. We refer the reader to the first chapters of [11] for more on the general theory.

Let $X / k$ be a proper smooth geometrically connected curve over a field and let $\pi: \mathscr{X} \rightarrow X$ be a $\boldsymbol{\mu}_{n^{-}}$ gerbe. If $L$ is an invertible sheaf on $\mathscr{X}$, then the natural map $\pi^{*} \pi_{*}\left(L^{\otimes n}\right) \rightarrow L^{\otimes n}$ is an isomorphism. This permits us to define a degree for invertible sheaves on the stack $\mathscr{X}$ (and by extension, if necessary, the degree of any coherent sheaf.)

Definition 1.2.1. Given an invertible sheaf $L$ on $\mathscr{X}$, the degree of $L$ is

$$
\operatorname{deg}(L)=\frac{1}{n} \operatorname{deg}_{X}\left(\pi_{*}\left(\mathscr{L}^{\otimes n}\right)\right) \in \mathbf{Q} .
$$

Given a locally free sheaf $V$ on $\mathscr{X}$, the slope of $V$ is $\mu(V)=\operatorname{deg}(\operatorname{det} V) / \operatorname{rk} V$.

Using the fact that every coherent sheaf on $\mathscr{X}$ has a finite resolution by locally free sheaves, one can extend the definition of degree and slope to arbitrary coherent sheaves. While this is entirely reasonable, it will not come up in the sequel.

Definition 1.2.2. A sheaf $V$ on $\mathscr{X}$ is stable (resp. semistable) if it is locally free and for all proper subsheaves $F \subsetneq V$ one has $\mu(F)<\mu(V)$ (resp. $\mu(F) \leq \mu(V)$ ).

Remark 1.2.3. It is easy to check that the condition that $\mu(F) \leq \mu(V)$ is the same as $\mu(V) \leq \mu(V / F)$. We will use this implicitly in the sequel.

One can similarly define the notion of geometrically (semi)stable. It turns out that semistability is a geometric property, while stability is not unless the sheaf in question is simple (see Example 1.3.9 and $\S 1.5$ of [11]). It is easy to see that one can test the property of (semi)stability by restricting attention solely to subsheaves $F \subset V$ for which the quotient $V / F$ is also locally free.

Definition 1.2.4. A semistable sheaf $V$ on $\mathscr{X}$ is (geometrically) polystable if $V$ (resp. $V \otimes \bar{k}$ ) is isomorphic to a direct sum of stable sheaves.

If $V$ is polystable, it follows from Remark 1.2.3 that the stable summands all have the same slope, and that this slope equals the slope of $V$. 
Remark 1.2.5. When the gerbe $\mathscr{X}$ is trivial, it is in general quite subtle to detect semistable sheaves. However, if $\mathscr{X}$ is non-trivial, then any locally free $\mathscr{X}$-twisted sheaf $V$ whose rank equals the index of the Brauer class attached to $\mathscr{X}$ is automatically stable. However, in this case it is quite subtle to detect when such a sheaf is geometrically stable. This issue will appear in a fundamental way in the analysis of section 2 .

Proposition 1.2.6. Given $\mu \in \mathbf{Q}$, the category of semistable $\mathscr{X}$-twisted sheaves of slope $\mu$ is an abelian category in which every object has finite length. The simple objects are the stable sheaves.

Proof. Let $\varphi: F \rightarrow G$ be a map of semistable sheaves of slope $\mu$. We will show that the kernel $K$ and cokernel $Q$ of $\varphi$ are both semistable of slope $\mu$. This will immediately prove the first statement by "transport of structure" from the abelian category of coherent sheaves on $\mathscr{X}$. We first claim that $Q$ is locally free. Indeed, we have

$$
\mu=\mu(F) \leq \mu(\varphi(F)) \leq \mu\left(\varphi(F)^{*}\right) \leq \mu(G)=\mu,
$$

where $\varphi(F)^{*}$ denotes the saturation of $\varphi(F)$ as a subsheaf of $G$. We conclude that $\varphi(F)=\varphi(F)^{*}$, so that $G / \varphi(F) \cong G / \varphi(F)^{*}$. It follows that $Q$ is locally free. Moreover, we have that the first two non-trivial terms in the sequence $0 \rightarrow \varphi(F) \rightarrow G \rightarrow Q \rightarrow 0$ have the same slope. It follows that $\mu(Q)=\mu$. To see that $Q$ is semistable, suppose $Q^{\prime} \subset Q$ is a subsheaf and let $G^{\prime}$ be the preimage of $Q^{\prime}$ in $G$, so that there is an exact sequence $0 \rightarrow \varphi(F) \rightarrow G^{\prime} \rightarrow Q^{\prime} \rightarrow 0$. By the semistability of $G$ we have that $\mu(\varphi(F)) \geq \mu\left(G^{\prime}\right)$, which implies that $\mu\left(G^{\prime}\right) \geq \mu\left(Q^{\prime}\right)$. Thus, $\mu\left(Q^{\prime}\right) \leq \mu$ and $Q$ is semistable.

It is immediate that $K$ is locally free. Since $\mu(F)=\mu(\varphi(F))$, we conclude that $\mu(K)=\mu(F)=\mu$. It now follows immediately from the semistable of $F$ that $K$ is semistable.

Corollary 1.2.7. If $\mathscr{F}$ is semistable of slope $\mu$ and $\left\{\mathscr{G}_{i}\right\}_{i \in I}$ is a set of stable subsheaves of $\mathscr{F}$ of slope $\mu$ then there is a subset $J \subset I$ such that $\Sigma_{i \in I} \mathscr{G}_{i}=\bigoplus_{j \in J} \mathscr{G}_{j}$ as subsheaves of $\mathscr{F}$.

Proof. Since $\mathscr{F}$ is Noetherian, we may assume that $I$ is finite. Consider the surjection $\bigoplus_{i} \mathscr{G}_{i} \rightarrow \Sigma_{i} \mathscr{G}_{i}$, and write $K$ for the kernel. Let $K^{\prime} \subset K$ be a stable subsheaf of slope $\mu$. The map $K^{\prime} \rightarrow \bigoplus \mathscr{G}_{i}$ is nonzero, which implies that one of the projections $K^{\prime} \rightarrow \mathscr{G}_{i_{0}}$ is an isomorphism. It follows that $\Sigma_{i} \mathscr{G}_{i}$ is the image of $\bigoplus_{i \neq i_{0}} \mathscr{G}_{i}$. By induction, $\Sigma_{i} \mathscr{G}_{i}$ is a direct sum of stable subsheaves.

The socle of a semistable sheaf $\mathscr{F}$ of slope $\mu$ is the sum of all of its stable subsheaves of slope $\mu$. It is clear that the socle is stable under all automorphisms of $\mathscr{F}$, and we have just shown that the socle is polystable.

Corollary 1.2.8. Let $k$ be a perfect field. Given a semisimple $\mathscr{X}$-twisted sheaf $\mathscr{F}$ of slope $\mu$, there is a canonical maximal subsheaf $S(\mathscr{F}) \subset \mathscr{F}$, compatible with base extension, such that $S(\mathscr{F})$ is geometrically polystable.

Proof. It follows from 1.2.6 that the sum $\Sigma \mathscr{G} \subset \mathscr{F} \otimes \bar{k}$, taken over all subsheaves $\mathscr{G} \subset \mathscr{F} \otimes k$ which are stable of slope $\mu$, is direct and stable under the operation of the absolute Galois group of $k$. Basic descent theory shows that this sheaf is the base extension of a subsheaf $S(\mathscr{F}) \subset \mathscr{F}$. Uniqueness is immediate.

1.3. Moduli of stable sheaves. Throughout this section, $X$ will be a smooth proper geometrically curve over a perfect field $k$. We recall from section 2.3 of [15] that the $\mathscr{X}$-twisted stable sheaves on $X$ of slope $\mu$ form an algebraic Deligne-Mumford stack which we denote by $\mathscr{M}_{\mathscr{X} / k}^{s, \mu}$. This is naturally a substack of $\underline{\mathrm{Spl}}_{\mathscr{X} / k}$. We denote its coarse moduli space by $\mathrm{M}_{\mathscr{X} / k}^{s, \mu}$ and note that $\mathscr{M}_{\mathscr{X} / k}^{s, \mu}$ is a $\mathbf{G}_{m}$-gerbe and may in fact be obtained as the pullback of the morphism $\underline{\mathrm{Spl}}_{\mathscr{X} / k} \rightarrow \mathrm{Spl}_{\mathscr{X} / k}$ via the inclusion $\mathrm{M}_{\mathscr{X} / k}^{s, \mu} \rightarrow \operatorname{Spl}_{\mathscr{X} / k}$.

We will now study the possible ranks of certain geometrically polystable sheaves. Given a geometrically polystable $\mathscr{X}$-twisted sheaf $V$ on $X$ of slope $\mu$, we have a decomposition $V \otimes \bar{k} \cong \bigoplus V_{i}$ with $V_{i}$ stable sheaves of slope $\mu$ on $X \otimes \bar{k}$. Each $V_{i}$ gives rise to a point $\left[V_{i}\right]$ on the coarse moduli space $\mathrm{M}_{\mathscr{X} / k}^{s, \mu}$ of stable $\mathscr{X}$-twisted sheaves of slope $\mu$. Write $I_{V}$ for the set of points $\left[V_{i}\right] \in \mathrm{M}_{\mathscr{X} / k}^{s, \mu}(\bar{k})$.

Lemma 1.3.1. With the above notation, there is a natural continuous action of $\mathrm{Gal}(k)$ on $I_{V}$. 
Proof. Write $V \otimes \bar{k}=\bigoplus_{i \in I_{V}} W_{i}$, where $W_{i}$ groups the stable summands with the given isomorphism class. Given an element $\sigma \in \operatorname{Gal}(k)$, the descent datum on $V \otimes \bar{k}$ induces an isomorphism $\bigoplus \sigma^{*} W_{i} \stackrel{\sim}{\rightarrow} \oplus W_{i}$. Since each $W_{i}$ (resp. $\sigma^{*} W_{i}$ ) is an isotypic and the $W_{i}$ (resp. $\sigma^{*} W_{i}$ ) have pairwise non-isomorphic stable constituents, it follows that there is an induced bijection $\widetilde{\sigma}: I_{V} \stackrel{\sim}{\rightarrow} I_{V}$ such that the isomorphism class associated to $\sigma^{*} W_{i}$ is the same as that associated to $W_{\widetilde{\sigma}(i)}$. The map $\sigma \mapsto \widetilde{\sigma}$ defines the action in question.

Lemma 1.3.2. In the notation of 1.3 .1 the sheaf $V$ is indecomposable if and only if the action of $\operatorname{Gal}(k)$ on $I_{V}$ is transitive.

Proof. The action of $\operatorname{Gal}(k)$ on an orbit of $I_{V}$ induces (via restriction) a descent datum on a proper sub-sum of the $W_{i}$. Taking the direct sum over all orbits yields a decomposition of $V$ as a direct sum indexed by orbits.

Lemma 1.3.3. Let $k$ be a field and $\alpha$ and $\beta$ two elements of $\operatorname{Br}(k)$. Suppose that for all field extensions $L / k$, we have that $\alpha_{L}=0$ if and only if $\beta_{L}=0$. Then $\alpha$ and $\beta$ generate the same cyclic subgroup of $\operatorname{Br}(k)$.

Sketch of proof. This is a well-known result of Amitsur. We provide a modern proof. Let $P$ be a BrauerSeveri variety with Brauer class $\alpha$. Since $\left.\alpha\right|_{P}=0$, we have that $\left.\beta\right|_{P}=0$. Examining the Leray spectral sequence in étale cohomology for $\mathbf{G}_{m}$ on the morphism $P \rightarrow$ Spec $k$, we see that the kernel of $\operatorname{Br}(k) \rightarrow \operatorname{Br}(P)$ is the subgroup generated by $\alpha$. Thus, $\beta \in\langle\alpha\rangle$. Reversing the roles of $\alpha$ and $\beta$ completes the proof.

Proposition 1.3.4. Given an indecomposable geometrically polystable $\mathscr{X}$-twisted sheaf $V$, there is a $\mu \in \mathbf{Q}$ and a closed point $p \in \mathrm{M}_{\mathscr{X} / k}^{s, \mu}$ such that the rank of $V$ is a multiple of $[\kappa(p): k] \operatorname{ind}(\alpha(p))$. Moreover, every such multiple is realized by an indecomposable geometrically polystable $\mathscr{X}$-twisted sheaf.

Proof. Write $V \otimes \bar{k}=\bigoplus V_{i}=\bigoplus W_{j}$ as above. By 1.3.2, the points [ $\left.V_{i}\right]$ form a single Galois orbit in $\mathrm{M}_{\mathscr{X} / k}^{s, \mu}(\bar{k})$. This corresponds to a closed point $p \in \mathrm{M}_{\mathscr{X} / k}^{s, \mu}$.

Consider the $k$-algebra $A:=\operatorname{End}(V)$. Tensoring with $\bar{k}$, we see that $A$ is a separable algebra. Since $V$ is indecomposable, it is easy to see that $A$ is in fact a division algebra. Thus, there is a finite extension $L$ of $k$ such that $A$ is a central division algebra over $L$. We claim that $L=\kappa(p)$ and that the class of $A$ in $\operatorname{Br}(L)$ equals the universal obstruction $\omega(p)$ restricted to $p$.

To prove that $L=\kappa(p)$, note that the set of idempotents of $A \otimes \bar{k}$ is in natural bijection with $I_{V}$, in a manner compatible with the action of $\operatorname{Gal}(k)$. It follows that the finite étale coverings $\operatorname{Spec} \kappa(p) \rightarrow \operatorname{Spec} k$ and $\operatorname{Spec} Z(A) \rightarrow$ Spec $k$ are isomorphic, which shows that $L \cong \kappa(p)$. To see that $[A]=\omega(p)$, it suffices to do so assuming that $k=L$. Indeed, the inclusion $L \subset \operatorname{End}(A)$ gives $V$ the structure of $X \otimes L$-module in such a way that $V \otimes_{L} \bar{L}$ is polystable and isotypic. Thus, we may assume that $A$ is a central division algebra over the base field and that $V \otimes_{k} \bar{k}$ is isotypic.

To show that $[A]=\omega(p)$, we first show that $[A]$ and $\omega(p)$ generate the same cyclic subgroup of $\operatorname{Br}(k)$. By 1.3.3, it suffices to show (upon extending the base field, which we will denote with $k$ by abuse of notation) that $A$ is split if and only if there is a stable bundle $V_{0}$ on $X$ with moduli point $p$. If $V_{0}$ exists, then we see that $V$ is an étale form of $V_{0}^{\oplus n}$ for some $n$. But Aut $\left(V_{0}^{\oplus n}\right)=\mathrm{GL}_{n, k}$, so by Hilbert's Theorem $90 V \cong V_{0}^{\oplus n}$. Thus, $A \cong \mathrm{M}_{n}(k)$. On the other hand, if $A$ is split then there is a full set of operators on $V$ splitting $V$ into a direct sum $V_{0}^{\oplus n}$ with $V_{0}$ geometrically stable. It follows that $\left[V_{0}\right]=p$ and thus that $\omega(p)=0$. (The reader will note that for the purposes of this paper, the equality of the cyclic subgroups $\langle[A]\rangle$ and $\langle\omega(p)\rangle$ is all that we need.)

Note that given any $V$, its forms are classified by $\mathrm{H}^{1}(\mathrm{Spec} k, \mathrm{GL}(A))$. By Hilbert's Theorem 90 for division algebras, this cohomology group is trivial. Thus, any two geometrically polystable geometrically isotypic $\mathscr{X}$-twisted sheaves with geometric summands supported at $p$ are isomorphic. Furthermore, the argument of the previous paragraph shows that the rank of $V$ is a multiple of $r$ ind $(\omega(p))$. Hence, to show that $[A]=\omega(p)$ it suffices to show that there is a $V$ of rank equal to $r$ ind $(\omega(p))$ whose endomorphism ring has Brauer class $\omega(p)$. Let $\xi$ be the residual gerbe at $p$ (the fiber of $\mathscr{M}_{\mathscr{X} / k}^{s, \mu} \rightarrow M_{\mathscr{X} / k}^{s, \mu}$ ). By definition, there is a $\xi$-twisted $\mathscr{X}$-twisted stable sheaf $\mathscr{V}$ on $\mathscr{X} \times \xi$ of rank $r$ with endomorphism ring $k$. If $F$ is a (-1)-fold $\xi$-twisted vector space, then $F \otimes \mathscr{V}$ is a geometrically polystable $\mathscr{X}$-twisted sheaf 
with geometric components supported at $p$. Furthermore, $\operatorname{End}(F \otimes \mathscr{V}) \cong \operatorname{End}(F)$ and this has Brauer class $\omega(p)$. The rank of $F$ is a multiple of ind $(\omega(p))$, and all multiples occur. Choosing one of minimal rank yields the desired result. (In fact, we have shown that any $V$ has the form $F \otimes \mathscr{V}$ for some $F$.)

\section{INDEX REDUCTION VIA STABLE TWISTED SHEAVES}

2.1. Twisted Riemann-Roch. We briefly summarize a twisted version of the Riemann-Roch theorem for gerbes. The reader is referred to section 2.2.7 of [15] for a proof. Let $f: X \rightarrow S$ be a proper lci morphism between quasi-projective varieties over a field. Let $\mathscr{S} \rightarrow S$ be a $\mu_{n}$-gerbe and let $\mathfrak{f}: \mathscr{X} \rightarrow \mathscr{S}$ be the pullback of $\mathscr{S}$ to $X$. Write $\mathrm{Td}_{f}$ for the relative Todd class of $f$ (the Todd class of the relative tangent complex). Let $\mathscr{F}$ be a coherent $\mathscr{X}$-twisted sheaf. For the purposes of this paper, define the rational Chow groups $A$ of $\mathscr{S}$ and $\mathscr{X}$ to be the rational Chow groups of $S$ and $X$, respectively. This is justified by the results of Vistoli's thesis [23]; one can check that there is a theory of Chern classes, etc., for this choice of Chow theory.

Proposition 2.1.1. There is an equality $\operatorname{ch}\left(\mathfrak{f}_{*} \mathscr{F}\right)=\mathfrak{f}_{*}\left(\operatorname{ch}(\mathscr{F}) \cdot \operatorname{Td}_{f}\right)$ in $A(\mathscr{S})$.

The case of primary interest to us will be when $S=\operatorname{Spec} k$ and $X$ is a curve. In this case, there is an ad hoc proof of the following corollary.

Corollary 2.1.2. Let $f: X \rightarrow$ Spec $k$ be a proper smooth curve and $\mathscr{S} \rightarrow$ Spec $k$ a $\boldsymbol{\mu}_{n}$-gerbe with pullback $\mathscr{X} \rightarrow X$. Write $\mathfrak{f}: \mathscr{X} \rightarrow \mathscr{S}$ for the projection. Given a coherent $\mathscr{X}$-twisted sheaf, the rank of the complex $\mathbf{R} \mathfrak{f}_{*} \mathscr{F}$ of $\mathscr{S}$-twisted vector spaces equals $\operatorname{deg}(\mathscr{F})+\operatorname{rk}(\mathscr{F})(1-g)$.

Proof. We give the proof in this case, as we will use it and it is simpler than the general case. One need only note that the formation of $\mathbf{R} \mathfrak{f}_{*}$ commutes with flat base change (Proposition 13.1.9 of [12]), and that there is a finite extension $L$ of $k$ and a flat map Spec $L \rightarrow \mathscr{S}$. Pulling back to $L$ reduces this to the classical Riemann-Roch formula.

Remark 2.1.3. The reasoning used in the proof of 2.1.2 also proves 2.1.1, but one must pay more attention to the properties of the Chow theory.

2.2. A formula for index reduction. In this section $X$ is a smooth proper geometrically connected curve over a perfect field $k$. Write $D$ for the index of $X$ (the g.c.d. of the degrees of all closed points) and $\delta$ for the index of $\operatorname{Pic}_{X / k}^{1}$. We clearly have that $\delta \mid D$ Let $\bar{\beta} \in \operatorname{Br}(k)$ be a Brauer class and $\beta \in \mathrm{H}^{2}\left(\operatorname{Spec} k, \boldsymbol{\mu}_{n}\right)$ a lift.

In the following, given a scheme $Y$, the notation " $p \in Y$ " will mean that $p$ is a closed point of $Y$. Given a Brauer class $\alpha \in \operatorname{Br}(Y)$ and $p \in Y$, we will write $\alpha(p)$ for $\left.\alpha\right|_{\operatorname{Spec} \kappa(p)} \in \operatorname{Br}(\kappa(p))$.

Definition 2.2.1. Given a scheme $Y$ and a Brauer class $\alpha \in \operatorname{Br}(Y)$, define the $\beta$-index reduction of $(Y, \alpha)$ by

$$
\iota_{\beta}(Y, \alpha)=\min _{p \in Y}[\kappa(p): k] \operatorname{ind}(\alpha(p)+\beta) .
$$

Given $r$ and $d$, write $\iota_{\beta}(r, d)$ for $\iota_{\beta}\left(M_{X / k}^{s}(r, d), \mathscr{M}_{X / k}^{s}(r, d)\right)$.

Proposition 2.2.2. The index of $\beta_{k(X)}$ is

$$
\operatorname{ind}\left(\beta_{k(X)}\right)=\min _{r \mid i, d \in[0, D)} r \iota_{\beta}(r, r d) .
$$

Furthermore, we have that

$$
\min _{d \in[0, D)} \iota_{\beta}(1, d) \text { divides } \delta \operatorname{ind}\left(\beta_{k(X)}\right) .
$$

The reader will note that the divisibility statement was originally proven by Schofield and Van den Bergh [22]. The present techniques give a new (but very closely related) proof of the result.

Let us set notation for the proof. Choose a $\boldsymbol{\mu}_{n}$-gerbe $\mathscr{S} \rightarrow$ Spec $k$ representing $\beta$. There is a $\boldsymbol{\mu}_{n}$-gerbe $\mathscr{X} \rightarrow X$ induced by pullback of $\mathscr{S}$. Write $i$ for the index of $\beta$ (over $k$ ). 
Proof. Given a locally free $\mathscr{X}$-twisted sheaf $V$ of minimal rank, we see that (1) $V$ is stable of some slope $\mu$, and (2) $V=S(V)$, the $\mu$-socle of $V$. It follows that we may assume that $V$ is geometrically polystable. By 1.3.4 and 1.1.6, we see that the index of $\beta_{k(X)}$, which is the rank of $V$, must equal the $\beta$ index reduction of $\left(\mathrm{M}_{X / k}^{s}, \mathscr{M}_{X / k}^{s}\right)$ at $p$ for some closed point $p \in \mathrm{M}_{X / k}^{s}$. It remains to show that the slope $\mu$ is integral, i.e., that $r \mid d$. To see this, let $\pi: \mathscr{X} \rightarrow \mathscr{S}$ denote the natural morphism. The RiemannRoch formula 2.1.2 shows that $\mathbf{R} \pi_{*} V$ is a complex of $\mathscr{S}$-twisted vector spaces of rank $d+r(1-g)$. We know that $i$ must divide this quantity, and that $r$ must divide $i$ (as the index cannot increase upon pullback!). By definition, $D$ is the minimal degree of a Cartier divisor on $X$. It follows that twisting $V$ by an appropriate invertible sheaf allows us to assume that $d$ lies between 0 and $D-1$, which proves the first statement.

To prove the second, suppose there is a $k$-rational point $q$ on $\operatorname{Pic}_{X / k}^{\delta}$. Thus, there is an $\alpha(q)$-twisted invertible sheaf $L$ of degree $\delta$. Applying 2.1.2 to the twisted sheaf $V \otimes L^{\otimes n}$ yields a complex of $\beta+n \alpha(q)$ twisted vector spaces of rank equal to $d+n r \delta+r(1-g)$. The gcd of these ranks divides $r \delta$, which almost yields the desired statement. To see that the gcd can be replaced by min (as the formula in this case requires), we use an argument essentially due to Schofield and van den Bergh. We may first replace $\beta$ by its $p$-primary component and assume that ind $\left(\beta_{k(X)}\right)$ is a power of $p$. (We implicitly use the fact that the set of $k$-rational Picard obstructions is a group in reassembling the result from its primary parts.) We may also replace $\delta$ by the largest $p$-power dividing it. Writing $d=r d^{\prime}$, the twisted Euler characteristic equals $r\left(d^{\prime}+n \delta+1-g\right)$. Choosing $n$ appropriately, we see that ind $(\beta+n \alpha(q))=r \delta^{\prime}$ with $0 \leq \delta^{\prime}<\delta$, so that ind $(\beta+n \alpha(q))<r \delta$. It follows that the index of the $p$-primary part must divide $r \delta$, as required. Furthermore, the $p$-primary part of $\beta+n \alpha(q)$ is $\beta+n \alpha(q)^{(p)}$, and it is easy to see that if $\alpha$ is a Picard obstruction then all of its primary parts are also Picard obstructions. The result follows.

This result has a particularly nice interpretation for curves of genus 1 (and 0 , although this case is already well known).

Corollary 2.2.3. If $g(X) \leq 1$ then $\operatorname{ind}\left(\beta_{k(X)}\right)=\min \left\{[E: k] \mid \beta_{X_{E}}\right.$ is split $\}$.

Remark 2.2.4. We may interpret this in a few different ways. For one, it says that if $D$ is a central division algebra over $k(X)$ representing the Brauer class $\beta_{k(X)}$, then $D$ has a maximal subfield of the form $E \otimes_{k} k(X)$ for $E / k$ finite. From another perspective, it says that we may reduce the computation of index reduction to an understanding of splitting: $\beta$ has index $m$ if and only if there is a finite extension $E / k$ of degree $m$ such that the class $\beta_{E}$ is split by the curve $X_{E}$.

We are able to derive from this a similar result in the case of an imperfect field:

Corollary 2.2.5. Let $k$ be an imperfect field of characteristic $p$ and suppose $p$ Xind $(\beta)$. If $g(X) \leq 1$ then $\operatorname{ind}\left(\beta_{k(X)}\right)=\operatorname{gcd}\left\{[E: k] \mid \beta_{X_{E}}\right.$ is split $\}$.

Proof. Let $F / k$ be the perfect closure of $k$. In other words, $F$ is a perfect field lying in a fixed algebraic closure $\bar{k}$ of $k$ which is closed under adjoining $p$ 'th roots of elements, and is a compositum of $p$-power extensions. By the assumption on the characteristic, we may find a finite extension $E / F$ of degree $i=\operatorname{ind}\left(\beta_{X_{F}}\right)$ such that $\beta_{X_{E}}$ is split. Since the condition of $\beta_{X_{E}}$ being split involves a finite set of equations with a finite number of elements of $E$, we may find a finitely generated subfield $E^{\prime} \subset E$ such that $\beta_{X_{E^{\prime}}}$ is split. Since $E / k$ is algebraic, we have $\left[E^{\prime}: k\right]$ is finite and must divide $i p^{l}$ for some $l \geq 0$. On the other hand, since $p \wedge n, \beta$ is split by a finite extension $L / k$ of degree prime to $p$. Therefore $\operatorname{gcd}\{[E: k],[L: k]\} \mid i$, forcing the desired conclusion.

Let us denote by $\alpha$ the Brauer class of the gerbe $\mathscr{P} i c_{X} \rightarrow \operatorname{Pic}_{X}$. To warm up to the proof of Corollary 2.2.3, we give the following lemma (which is actually a special case):

Lemma 2.2.6. The class $\beta_{k(X)}$ is trivial if and only if $\beta=\alpha(p)$ for some point $p \in \operatorname{Pic}_{X}(k)$.

Proof. We note that $\beta_{k(X)}$ is trivial if and only if there is a $\beta$-twisted invertible sheaf on $X$, or in other words, if there is an object in $\mathscr{P}_{i c} \mathscr{X}(k)$. By[1.1.7, we have an identification Pic $\mathscr{X}=\mathrm{Pic}_{X}$ and using this, the Brauer class of the gerbe $\mathscr{P}_{i C_{\mathscr{X}}} \rightarrow \mathrm{Pic}_{\mathscr{X}} \cong \mathrm{Pic}_{X}$ is given by $\alpha+\beta$. Therefore, noting that having an object of $\mathscr{P}_{i c} \mathscr{X}(k)$ is equivalent to having a point in $p \in \operatorname{Pic}_{X}(k)$ with trivial obstruction $\alpha(p)+\beta$, this says $\beta_{k(X)}$ is split if and only if there is a $p \in \operatorname{Pic}_{X}(k)$ with $\alpha(p)=-\beta$. 
To finish, we note that since the kernel $\operatorname{Br}(k) \rightarrow \operatorname{Br}(k(X))$ is a subgroup, $\beta_{k(X)}$ is split if and only if $-\beta_{k(X)}$ is split. The above argument then shows this to be equivalent to the existence of a $p \in \operatorname{Pic}_{X}(k)$ with $\alpha(p)=\beta$.

Proof of Corollary 2.2.3 Using the fact that any stable vector bundle on a genus 1 curve with $r \mid d$ is invertible ([2]), and any stable vector bundle on a genus 0 curve is invertible, it follows from 2.2 .2 that we have

$$
\begin{aligned}
\operatorname{ind}\left(\beta_{k(X)}\right) & =\min _{d \in[0, \delta)} \iota_{\beta}(1, d) \\
& =\min _{d}\left\{\min \left\{[k(p): k] \operatorname{ind}(\beta+\alpha(p)) \mid p \in \operatorname{Pic}_{X}^{d}\right\}\right\} \\
& =\min \left\{[k(p): k] \operatorname{ind}(\beta+\alpha(p)) \mid p \in \operatorname{Pic}_{X}\right\} \\
& =\min \left\{[L: k] \operatorname{ind}(\beta+\alpha(p)) \mid p \in \operatorname{Pic}_{X}(L)\right\} \\
& =\min \left\{[L: k] \operatorname{ind}\left(\beta_{L}+\gamma\right) \mid \gamma \in \operatorname{Br}\left(X_{L} / L\right)\right\} \quad \text { (by Lemma2.2.6) } \\
& =\min \left\{[L: k] \operatorname{ind}\left(\beta_{L}-\gamma\right) \mid \gamma \in \operatorname{Br}\left(X_{L} / L\right)\right\} \\
& =\min \left\{[L: k][E: L] \mid \beta_{E} \sim \gamma_{E}, \gamma \in \operatorname{Br}\left(X_{L} / L\right)\right\} \\
& =\min \left\{[E: k] \mid \beta_{X_{E}} \text { is split }\right\}
\end{aligned}
$$

2.3. Higher-dimensional varieties. We indicate in this section how to extend our results to higher dimensional varieties over $k$. For the most part, the results are straightforward generalizations of the techniques above. One must do slightly more numerical work with the Riemann-Roch formula (as in [22]). There is also a slight complcation coming from the difference between the category of torsion free sheaves and its quotient by the category of sheaves supported in codimension at least 2 (which is necessary to mimic the argument reducing to the geometrically polystable case). We are content to simply state the results and leave the mostly straightforward details to the reader.

Let $X$ be a smooth geometrically connected projective variety over $k$ of dimension $t$ with fixed ample invertible sheaf $\mathscr{O}(1)$. Any section $\sigma$ of $\operatorname{Pic}_{X / k}$ has a well-defined degree given by the top selfintersection of a divisor on $X \otimes \bar{k}$ representing $\sigma$. Since the degree is an intersection-theoretic invariant, it is clearly constant on connected components of $\mathrm{Pic}_{X / k}$. We will write $\mathscr{P}_{i c_{X / k}^{d}}$ for the stack of invertible sheaves of degree $d$; the usual results show that $\mathscr{P}_{i} c_{X / k}^{d}$ is a $\mathbf{G}_{m}$-gerbe over its sheafification $\operatorname{Pic}_{X / k}^{d}$.

Let $\delta$ be the gcd of the degrees of all $k$-rational sections of $\mathrm{Pic}_{X / k}$ and let $D$ be the gcd of the degrees of all $k$-rational sections of $\mathscr{P} i_{X / k}$ (i.e., those sections of $\mathrm{Pic}_{X / k}$ which arise from actual invertible sheaves on $X$ ).

Definition 2.3.1. Given a torsion free coherent sheaf $\mathscr{E}$ of rank $r$ on $X$, the generalized slope of $\mathscr{E}$ is $\chi(\mathscr{E}) / r$.

Recall that a torsion free sheaf $\mathscr{E}$ of positive rank $r$ on $X$ is slope semistable (resp. stable) with respect to $\mathscr{O}(1)$ if for all proper subsheaves $\mathscr{F} \subset \mathscr{E}$ with strictly smaller rank, we have that $c_{1}(\mathscr{F}) / \operatorname{rk}(\mathscr{F})-c_{1}(\mathscr{E}) / r$ has non-positive (resp. strictly negative) intersection with $\operatorname{dim}(X)-1$ copies of a divisor in $|\mathscr{O}(1)|$. It is a standard result that the stack of slope semistable sheaves is an Artin stack with the substack of slope stable sheaves an open substack which is a $\mathbf{G}_{m}$-gerbe over an algebraic space. We will denote the stack of slope stable torsion free sheaves of rank $r$ and generalized slope $\mu$ by by $\mathscr{M}_{X / k}^{s}(r, \mu)$ and its sheafification by $M_{X / k}^{s}(r, \mu)$.

Let $\beta \in \operatorname{Br}(k)$ have index $i$. Let $\mathscr{S} \rightarrow$ Spec $k$ be a $\boldsymbol{\mu}_{n}$-gerbe representing $\beta$ and $\mathscr{X}=X \times_{k} \mathscr{S}$. We will write $\mathfrak{f}: \mathscr{X} \rightarrow \mathscr{S}$ for the natural map. Given an $\mathscr{X}$-twisted sheaf $\mathscr{F}$, we will write $\chi(\mathscr{F})$ for the rank of $\mathbf{R} \mathfrak{f}_{*} \mathscr{F}$ (as a complex of $\mathscr{S}$-twisted sheaves).

Proposition 2.3.2. The index of $\beta_{k(X)}$ is

$$
\operatorname{ind}\left(\beta_{k(X)}\right)=\min _{r \mid i, \mu \in \mathbf{Z}} r \iota_{\beta}\left(M_{X / k}^{s}(r, \mu), \mathscr{M}_{X / k}^{s}(r, \mu)\right) .
$$


Furthermore, we have that

$$
\min _{d \in[0, D)} \iota_{\beta}\left(\operatorname{Pic}_{X / k}^{d}, \mathscr{P} i c_{X / k}^{d}\right) \text { divides } \delta \operatorname{ind}\left(\beta_{k(X)}\right) .
$$

As above, the second statement is (for higher dimensional varieties) a refinement of the main result of Schofield and Van den Bergh: they consider only very ample sections of Pic when computing $\delta$, while an argument in the derived category as above shows that in fact one can strengthen the result (i.e., lower $\delta$ ) by considering arbitrary sections of Pic.

The proof of 2.3 .2 follows precisely the outline of the proof of 2.2 .2 above. We sketch the proof of the second statement, following p. 732 of [22] essentially verbatim as an aid to the reader. We may assume that $\beta$ is $p$-primary for some prime $p$, so that we may assume that $\delta$ is realized by a $k$-point $q$ of of $\operatorname{Pic}_{X / k}$, corresponding to some $\alpha(q)$-twisted invertible sheaf $\mathscr{L}$. Let $V$ be a torsion free $\mathscr{X}$-twisted sheaf of rank ind $\left(\beta_{k(X)}\right)$. It follows from 2.1.1 that

$$
\chi\left(V \otimes \mathscr{L}^{\otimes m}\right)=\frac{\delta \operatorname{ind}\left(\beta_{k(X)}\right)}{t !} m^{t}+\text { lower order terms. }
$$

Standard manipulations of numerical polynomials show that for a fixed $m$, we have

$$
\delta \operatorname{ind}\left(\beta_{k(X)}\right)=\sum_{j=0}^{t}(-1)^{j}\left(\begin{array}{l}
t \\
j
\end{array}\right) \chi\left(V \otimes \mathscr{L}^{m+t-j}\right) .
$$

Since $\delta=p^{a}$ and $\operatorname{ind}\left(\beta_{k(X)}\right)=p^{b}$ for some $a$ and $b$, it follows that there is some $N$ such that $\chi\left(V \otimes \mathscr{L}^{N}\right)$ is divisible by at most $p^{a+b}$. This is easily seen to imply the $p$-primary part of the required statement. The general case follows by reassembling the primary parts.

\section{EXAMPLES}

3.1. Index reduction for local fields. We may use Corollary 2.2 .3 to give precise information about index reduction in the case of local fields, using the work of Roquette [21] which computes the relative Brauer group of a curve in this case.

Let $k$ be a local field, and let $C / k$ be a curve (which will soon be assumed to have genus 1 ). We recall the following result:

Theorem 3.1.1 ([21], Theorem 1). Let $A$ be a central simple $k$ algebra. Then $A \otimes_{k} k(C)$ is split if and only if ind $(A) \mid \operatorname{ind}(C)$.

By standard facts from local class field theory, we know that for $E / k$ finite,

$$
\operatorname{ind}\left(A_{E}\right)=\operatorname{ind}(A) / \operatorname{gcd}\{\operatorname{ind}(A),[E: k]\} .
$$

Now suppose that $C$ has genus 1 , and write $i=\operatorname{ind}(A)$. We may rewrite Corollary 2.2.3 as saying:

$$
\operatorname{ind}\left(A_{k(C)}\right)=\operatorname{gcd}\left\{[E: k]\left|\frac{i}{\operatorname{gcd}\{i,[E: k]\}}\right| \operatorname{ind}\left(C_{E}\right)\right\} .
$$

The computation of the index of $A_{k(C)}$ may therefore be expressed entirely in terms of arithmetic information about the curve $C$, in particular, how to compute its index over different finite extensions. To give an example of this, let us consider the case where the index of $C$ is $p$, a prime number, and suppose $\operatorname{ind}(A)=m p^{n}$, where $p \nmid \backslash m, n>0$. For such a curve $C$, we define its capacity $\operatorname{cap}(C)$ as:

$$
\operatorname{cap}(C)=\max \left\{r \mid \begin{array}{c}
\exists L / k \text { a finite field extension with } \\
{[L: k]=m^{\prime} p^{r}, p \quad \backslash m^{\prime}, \text { and } C(L)=\emptyset}
\end{array}\right\} .
$$

We then have:

$$
\operatorname{ind}\left(A_{k(C)}\right)=\left\{\begin{array}{cl}
m p^{n} & \text { if } \operatorname{cap}(C)<n-1 \\
m p^{n-1} & \text { if } \operatorname{cap}(C) \geq n-1
\end{array}\right.
$$


3.2. Counterexamples for non-constant classes. It is natural to ask whether or not the formula of Corollary 2.2.3 holds for arbitrary unramified Brauer classes on genus 1 curves as well as constant classes. In other words, is it possible that for every $\beta \in \operatorname{Br}(X)$ where $X$ is a genus 1 curve, $\operatorname{ind}\left(\beta_{k(X)}\right)$ is equal to $\operatorname{gcd}\left\{[E: k] \mid \beta_{E}\right.$ is split\}? In this section we give counterexamples to this in the case where $X$ is elliptic (i.e., $X(k) \neq \emptyset$ ).

To construct our counterexamples, we must start with a few generalities about genus 1 curves and Brauer classes on their Jacobians. Let $\mathcal{C}$ a genus 1 curve over $k$, and $\mathcal{E}=\operatorname{Pic}^{0} \mathcal{C}$ its Jacobian. Let $\gamma \in \mathrm{H}^{1}(k, \mathcal{E})$ be a cocycle representing $\mathcal{C}$. Recall from the Hocschild-Serre spectral sequence

$$
\mathrm{H}^{p}\left(k, \mathrm{H}^{q}\left(\mathcal{E}_{\bar{k}}, \mathbf{G}_{m}\right)\right) \Longrightarrow \mathrm{H}^{p+q}\left(\mathcal{E}, \mathbf{G}_{m}\right),
$$

we have an exact sequence:

$$
0 \rightarrow \operatorname{Br}(k) \rightarrow \operatorname{Br}(\mathcal{E}) \rightarrow \mathrm{H}^{1}(k, \mathcal{E}) \rightarrow 0
$$

Since $\mathcal{E}$ is has a canonical point (its origin), we may split the above exact sequence, and we obtain an isomorphism $\mathcal{A}: \mathrm{H}^{1}(k, \mathcal{E}) \stackrel{\sim}{\rightarrow} \operatorname{br}(\mathcal{E})$, where $\operatorname{br}(\mathcal{E})$ is defined to be those Brauer classes which specialize trivially at the origin of $\mathcal{E}$. We may therefore associate to our curve $\mathcal{C}$ a Brauer class $\mathcal{A}_{\mathcal{C}}$ on $\mathcal{E}$.

Remark 3.2.1. Since this is a group isomorphism, we have $\operatorname{per}(\mathcal{C})=\operatorname{per}\left(\mathcal{A}_{\mathcal{C}}\right)$. Further, the isomorphism is functorial with respect to extensions of the ground field. In other words, we have a natural isomorphism of functors from the category of field extensions of $k$ to the category of abelian groups defined by

$$
L / k \mapsto \mathrm{H}^{1}\left(L, \mathcal{E}_{L}\right),
$$

and

$$
L / k \mapsto \operatorname{br}\left(\varepsilon_{L}\right),
$$

respectively. This functoriality tells us that the index of $\mathcal{C}$ may be computed as

$$
\operatorname{ind}(\mathcal{C})=\operatorname{gcd}\left\{[E: k] \mid \mathcal{A}_{\mathfrak{C}} \otimes_{k} E \text { is split }\right\} .
$$

Proposition 3.2.2. Choose a complex elliptic curve $E /$ C. Let $k=\mathbf{C}(t)$ and let $\mathcal{E}=E \times_{\operatorname{Spec}(\mathbf{C})} \operatorname{Spec}(k)$. Then for any integer $n$ there exists a principal homogeneous space for $\mathcal{E}$ such that $\operatorname{ind}\left(\mathcal{A}_{\mathfrak{C}}\right)=n$ and $\operatorname{gcd}\left\{[E: k] \mid \mathcal{A}_{\mathcal{C}} \otimes_{k} E\right.$ is split $\}=n^{2}$.

Proof. Suppose $\gamma \in \mathrm{H}^{1}(k, \mathcal{E})_{n}$ is a nonzero class of order $n$ corresponding to a homogeneous space C. Since the corresponding algebra $\mathcal{A}_{\mathcal{C}}$ at the generic point of $\mathcal{E}$ is a central simple algebra over the function field of an algebraic surface, by the theorem of de Jong ([9]), we have

$$
n=\operatorname{per}(\mathcal{C})=\operatorname{per}\left(\mathcal{A}_{\mathfrak{C}}\right)=\operatorname{ind}\left(\mathcal{A}_{\mathcal{C}}\right) .
$$

By Remark 3.2.1, in order to complete the proof, it suffices to find $\mathcal{C}$ such that ind $(\mathcal{C})=\operatorname{per}(\mathcal{C})^{2}$. In order to calculate the index of $\mathcal{C}$, we examine the Kummer sequence

$$
0 \rightarrow \mathcal{E}_{n} \rightarrow \mathcal{E} \stackrel{n}{\longrightarrow} \mathcal{E} \rightarrow 0 .
$$

From the long exact sequence in cohomology, we find an exact sequence

$$
0 \rightarrow \mathcal{E}(k) / n \mathcal{E}(k) \rightarrow \mathrm{H}^{1}\left(k, \varepsilon_{n}\right) \rightarrow \mathrm{H}^{1}(k, \mathcal{E})_{n} \rightarrow 0 .
$$

We now calculate that

$$
\begin{aligned}
\mathcal{E}(k)=\operatorname{Hom}_{k}(\operatorname{Spec} k, \mathcal{E})=\operatorname{Hom}_{\mathbf{C}(t)}(\operatorname{Spec}(\mathbf{C}(t)), \mathcal{E}) & \\
=\operatorname{Hom}_{\mathbf{C}(t)}\left(\mathbf{C}(t), E \times_{\operatorname{Spec}(\mathbf{C})}\right. & \operatorname{Spec}(\mathbf{C}(t))) \\
& =\operatorname{Hom}_{\mathbf{C}}(\operatorname{Spec}(\mathbf{C}(t)), E)=\operatorname{Hom}_{\mathbf{C}}\left(\mathbf{P}^{1}, E\right) .
\end{aligned}
$$

Since every morphism from $\mathbf{P}^{1}$ to an elliptic curve is constant, this says

$$
\mathcal{E}(k)=\operatorname{Mor}_{\mathbf{C}}\left(\mathbf{P}^{1}, E\right)=E(\mathbf{C}),
$$

and in particular, $\mathcal{E}(k)$ is a divisible group. Therefore, $\mathcal{E}(k) / n \mathcal{E}(k)=0$, and the exact sequence 1 gives an isomorphism

$$
\mathrm{H}^{1}\left(k, \mathcal{E}_{n}\right)=\mathrm{H}^{1}(k, \mathcal{E})_{n} .
$$


Since $\mathbf{C} \subset k$, all the torsion points of $\mathcal{E}$ are defined over $k$, and this gives an isomorphism

$$
\mathrm{H}^{1}(k, \mathcal{E})_{n} \cong \operatorname{Hom}(\operatorname{Gal}(k), \mathbf{Z} / n \mathbf{Z})^{2} .
$$

Let $\varphi_{1}, \varphi_{2}: \operatorname{Gal}(k) \rightarrow \mathbf{Z} / n \mathbf{Z}$ surjective homomorphisms such that the corresponding cyclic extensions $L_{i}$ of $k$ are linearly disjoint. It is easy to see that the cohomology class corresponding to $\varphi_{i}$ is split by a field extension $F / k$ if and only if there is a map of $k$-algebras $L_{i} \rightarrow F$. Thus, any field $F$ which splits both $\varphi_{1}$ and $\varphi_{2}$ must have degree divisible by $n^{2}$, and in fact $F=L_{1} \otimes L_{2}$ is such a field of minimal degree. On the other hand, $\mathcal{C}$ has a point over $F$ if and only if both $\varphi_{1}$ and $\varphi_{2}$ are split by $F$. We conclude that ind $(\mathcal{C})=n^{2}=\operatorname{per}(\mathcal{C})^{2}$, as desired.

\section{INDEX REDUCTION ON TORSORS UNDER ABELIAN VARIETIES AND HOMOGENEOUS TWISTED BUNDLES}

In this section we sketch an approach to index reduction on torsors under abelian varieties using twisted Fourier-Mukai transforms. As we show, there is a connection between the existence of homogeneous minimal bundles and an index reduction formula involving only the universal Picard obstruction. Such homogeneity results are perhaps analogous to the explicit vector bundles used in the index reduction formulas arising in [19, 16] for certain homogeneous spaces under linear algebraic groups using the $K$-theory of such spaces. The main result of this section may be interpreted as evidence that homogeneous index reduction is exceedingly unlikely for torsors under abelian varieties. Since the $K$-theory of such a torsor is not generated by equivariant sheaves (in contrast to the rational case), this should not be surprising.

4.1. Twisted Fourier-Mukai transforms. In this section we prove a twisted form of Mukai's theorem on derived equivalences of abelian varieties, relating twisted sheaves on an $A$-torsor to twisted sheaves on $A$ in a certain Brauer class. For the sake of simplicity, we restrict our attention to bounded derived categories of twisted sheaves.

Let $k$ be a field and $A$ an abelian variety over $k$ and $T$ an $A^{\vee}$-torsor. Suppose $\beta \in \operatorname{Br}(k)$ is a Brauer class and let $\mathscr{T} \rightarrow T$ be a $\mathbf{G}_{m}$-gerbe representing $\beta_{T}$.

Definition 4.1.1. The twisted Picard stack parametrizing invertible $\mathscr{T}$-twisted sheaves will be denoted $\mathscr{P}_{i C_{T} / k}$.

Applying 1.1.7, we see that $\mathscr{P} i c_{\mathscr{T} / k}$ is a $\mathbf{G}_{m}$-gerbe over $\mathrm{Pic}_{T / k}$ with Brauer class $\left[\mathscr{P}_{i c_{T / k}}\right]+\beta$. In particular, there is a distinguished connected component $\mathscr{P}_{i c_{\mathscr{T} / k}^{0}}^{0}$ corresponding to the component of $\mathscr{P}_{i} c_{T / k}$ containing the point $\mathscr{O}_{T}$. It follows that $\mathscr{P}_{i c^{T} / k}^{0}$ is a $\mathbf{G}_{m}$-gerbe over $A$, such that the fiber over the identity section, viewed as a $\mathbf{G}_{m}$-gerbe, has Brauer class $\beta$. We will write $\mathscr{A} \rightarrow A$ for this gerbe in what follows (the class $\beta$ being understood throughout).

There is a universal invertible sheaf $\mathscr{L}$ on $\mathscr{A} \times \mathscr{T}$; the geometric fibers of $\mathscr{L}$ over $\mathscr{T}$ are $\mathscr{A}$-twisted, while the geometric fibers over $\mathscr{A}$ are $\mathscr{T}$-twisted. We can thus define a Fourier-Mukai transform $\Phi: \mathbf{D}_{-1}^{b}(\mathscr{A}) \rightarrow \mathbf{D}_{1}^{b}(\mathscr{T})$ with kernel $\mathscr{L}$ from the derived category of (-1)-fold $\mathscr{A}$-twisted coherent sheaves to the derived category of coherent $\mathscr{T}$-twisted sheaves.

Proposition 4.1.2. The functor $\Phi$ is an equivalence of categories.

Proof. By an argument formally identical to Lemma 2.12 of [18], it suffices to prove the statement when $k$ is algebraically closed. In this case, $\mathscr{T} \rightarrow T$ and $\mathscr{A} \rightarrow A$ are trivial gerbes. Given a trivialization of $\mathscr{T} \rightarrow T$ and a $k$-point of $T$, one naturally gets an identification $T \stackrel{\sim}{\rightarrow} A^{\vee}$ and a trivialization of $\mathscr{A} \rightarrow A$ (e.g., by the standard method of "rigidifications" of invertible sheaves [3]). Moreover, trivializations of $\mathscr{T} \rightarrow T$ and $\mathscr{A} \rightarrow A$ serve to identify the derived categories of $n$-fold $\mathscr{T}$-twisted (resp. $\mathscr{A}$-twisted) sheaves with coherent sheaves on $T$ (resp. $A$ ) for any $n$. It is easy to check that composing $\Phi$ with these equivalences yields the standard Fourier-Mukai transform $\mathbf{D}^{b}(A) \rightarrow \mathbf{D}^{b}\left(A^{\vee}\right)$, which is an equivalence by Theorem 2.2 of [17]. 
4.2. Moduli of homogeneous bundles. Using the twisted Fourier-Mukai transform, we can describe certain moduli spaces of locally free twisted sheaves on $T$ in terms of moduli spaces of finite length twisted sheaves on $\mathscr{A}$.

Let $\beta \in \operatorname{Br}(k)$, and let $\mathscr{A} \rightarrow A$ and $\mathscr{T} \rightarrow T$ be as above. Note that in constructing $\mathscr{T} \rightarrow T$ we may explicitly use the 1-fibered product of $T$ with a $\mathbf{G}_{m}$-gerbe over Spec $k$ representing $\beta$. It follows that $A$ acts on $\mathscr{T}$ functorially (and not merely pseudo-functorially). This allows us to think about the pullback of a $\mathscr{T}$-twisted sheaf via a translation by a point of $A$ in a very concrete manner; we will implicitly do this in what follows.

We will write $p$ for the first projection $A \times T \rightarrow A$, $q$ for the second projection $A \times T \rightarrow T$, and $\mu$ for the action $A \times T \rightarrow T$.

Definition 4.2.1. A $\mathscr{T}$-twisted sheaf $\mathscr{F}$ is

(1) homogeneous if $\tau_{x}^{*} \mathscr{F}_{\kappa(x)} \cong \mathscr{F}_{\kappa(x)}$ for all geometric points $x$ : Spec $\kappa \rightarrow A$;

(2) uniformly homogeneous if the scheme $\operatorname{Isom}_{A}\left(\mu^{*} \mathscr{F}, q^{*} \mathscr{F}\right) \rightarrow A$ has sections étale-locally on $A$.

Note that uniformly homogeneous sheaves are automatically homogeneous, and that homogeneous sheaves are locally free. The classical literature deals with abelian varieties over algebraically closed fields, and the usual definition of homogeneity works only with geometric points with coefficients in the base field.

Lemma 4.2.2. A $\mathscr{T}$-twisted sheaf $\mathscr{F}$ is homogeneous if and only if $\tau_{x}^{*} \mathscr{F}_{\kappa(x)} \cong \mathscr{F}_{\kappa(x)}$ for arbitrary fieldvalued (not necessarily geometric) points $x: \operatorname{Spec} \kappa \rightarrow A$.

Proof. Suppose $\mathscr{F}$ is homogeneous. Without loss of generality, we may assume $\kappa(x)=k$. By the compatibility of cohomology with flat base change, the sheaf of isomorphisms $\operatorname{Isom}\left(\tau_{x}^{*} \mathscr{F}, \mathscr{F}\right)$ is represented by an open subscheme of the affine space $\operatorname{Hom}\left(\tau_{x}^{*} \mathscr{F}, \mathscr{F}\right)$; this subscheme is non-empty by the homogeneity assumption. Thus, if $k$ is infinite, it immediately follows that there is a rational point. If $k$ is finite, then we need only note that the Isom scheme is a torsor under the automorphism sheaf $\mathscr{A} u t(\mathscr{F})$, which is a smooth geometrically connected group scheme over $k$ (see 4.2.4 below). By Lang's theorem, any torsor has a rational point.

Corollary 4.2.3. Given a field extension $K / k$, a $\mathscr{T}$-twisted sheaf $\mathscr{F}$ is homogeneous if and only if $\mathscr{F} \otimes K$ is homogeneous.

Given two sheaves $F$ and $G$ on $T \times A$, define a functor $\underline{\operatorname{Hom}}_{A}(F, G)$ on the category of $A$ schemes by sending $\gamma: S \rightarrow A$ to $\operatorname{Hom}_{S}\left(F_{S}, G_{S}\right)$.

Lemma 4.2.4. The scheme $\operatorname{Isom}_{A}\left(\mu^{*} \mathscr{F}, q^{*} \mathscr{F}\right) \rightarrow A$ The functor $\underline{\operatorname{Hom}}_{A}\left(\mu^{*} \mathscr{F}, q^{*} \mathscr{F}\right)$ is represented by a closed cone (with linear geometric fibers) in a geometric vector bundle and contains $\operatorname{Isom}_{A}\left(\mu^{*} \mathscr{F}, q^{*} \mathscr{F}\right)$ as an open subscheme.

Proof. Let $\mathscr{W}$ be a locally free $\mathscr{T}$-twisted sheaf and $\mathscr{O}(1)$ an ample invertible sheaf on $T$. Choose $N$ and $m$ such that there is a surjection $\pi: \mathscr{W}(N)^{m} \rightarrow \mu^{*} \mathscr{F}$ and such that $\mathbf{R}^{i} p_{*} \mathscr{H} o m\left(\mathscr{W}, q^{*} \mathscr{F}\right)(N)=0$ for $i>0$. Writing $\mathscr{K}$ for the kernel of $\pi$, we may similarly choose $N^{\prime} \geq N$ and $m^{\prime}$ such that there is a surjection $\mathscr{W}\left(N^{\prime}\right)^{m^{\prime}} \rightarrow \mathscr{K}$. There results a complex

$$
\mathscr{W}\left(N^{\prime}\right)^{m^{\prime}} \rightarrow \mathscr{W}(N)^{m} \rightarrow \mu^{*} \mathscr{F} \rightarrow 0 .
$$

The cohomological assumptions show that there is a geometric vector bundle $\mathbf{V}$ (resp. $\mathbf{V}^{\prime}$ ) whose sheaf of sections is

$$
p_{*}\left(\mathscr{H} o m\left(\mathscr{W}, \mu^{*} \mathscr{F}\right)(N)^{m}\right)
$$

(resp. $\left.p_{*}\left(\mathscr{H} o m\left(\mathscr{W}, \mu^{*} \mathscr{F}\right)\left(N^{\prime}\right)^{m^{\prime}}\right)\right)$. Moreover, the presentation of $\mu^{*} \mathscr{F}$ yields an exact sequence of abelian sheaves

$$
0 \rightarrow \underline{\operatorname{Hom}}_{A}\left(\mu^{*} \mathscr{F}, q^{*} \mathscr{F}\right) \rightarrow \mathbf{V} \rightarrow \mathbf{V}^{\prime},
$$

where the latter map is linear. It follows that $\operatorname{Hom}_{A}\left(\mu^{*} \mathscr{F}, q^{*} \mathscr{F}\right)$ is represented by a closed cone in $\mathbf{V}$ with linear fibers. That isomorphisms form an open subscheme is immediate.

Proposition 4.2.5. A $\mathscr{T}$-twisted sheaf $\mathscr{F}$ is homogeneous if and only if it is uniformly homogeneous. 
Proof. Suppose $\mathscr{F}$ is homogeneous, so that the open subscheme

$$
\operatorname{Isom}_{A}\left(\mu^{*} \mathscr{F}, q^{*} \mathscr{F}\right) \subset \underline{\operatorname{Hom}}_{A}\left(\mu^{*} \mathscr{F}, q^{*} \mathscr{F}\right)
$$

meets every fiber. In this case, since isomorphisms are dense in every fiber (the fibers being geometrically integral) and each fiber is a torsor under $\operatorname{Aut}(\mathscr{F})$, we see that the fibers of $\underline{\operatorname{Hom}}_{A}\left(\mu^{*} \mathscr{F}, q^{*} \mathscr{F}\right)$ are all of the same dimension. Thus, the linear map $\mathrm{V} \rightarrow \mathrm{V}^{\prime}$ from the proof of 4.2 .4 has constant rank in every geometric fiber. Since $A$ is reduced, we conclude that the kernel is a subbundle of $\mathbf{V}$, i.e., that $\underline{\operatorname{Hom}}_{A}\left(\mu^{*} \mathscr{F}, q^{*} \mathscr{F}\right)$ is represented by a geometric vector bundle, hence is smooth over $A$. As an open subscheme hitting every fiber, $\operatorname{Isom}_{A}\left(\mu^{*} \mathscr{F}, q^{*} \mathscr{F}\right)$ must have sections everywhere étale-locally over $A$.

The other direction is immediate.

Definition 4.2.6. A flat family of homogeneous $\mathscr{T}$-twisted coherent sheaves parametrized by $S$ is an $S$-flat quasi-coherent $\mathscr{T}$-twisted sheaf of finite presentation $\mathscr{F}$ on $\mathscr{T} \times S$ such that for all geometric points $s \rightarrow S$, the fiber $\mathscr{F}_{s}$ is homogeneous.

It is clear that the collection of flat families of homogeneous coherent $\mathscr{T}$-twisted sheaves forms a stack. We will prove in a moment that it is in fact a quasi-proper Artin stack, although this is not immediately obvious. It is quite easy to see that it is a constructible substack of the stack of coherent $\mathscr{T}$-twisted sheaves (left to the reader), but we are not even sure if it is locally closed. (It is unlikely to be closed, as any homogeneous sheaf is locally free.)

We beg the reader's indulgence in allowing us the following notational convenience.

Convention 4.2.7. Given a scheme $S$ and a stack $\mathscr{S}$ of categories on the fppf site of $S$, we will say that " $\mathscr{S}$ is an Artin stack" (etc.) if the underlying stack of groupoids is an Artin stack. Thus, when we talk about stacks of sheaves, we include all morphisms in the category structure of the fiber categories, throwing away all but the isomorphisms only when we are considering the algebraicity properties of the stack in question.

Definition 4.2.8. The stack of homogeneous $\mathscr{T}$-twisted sheaves of rank $r$ will be denoted $\mathbf{H}_{\mathscr{T} / k}^{r}$.

There is another stack which will be of interest in this section.

Definition 4.2.9. Given an Artinian $\mathscr{A}$-twisted coherent sheaf $\mathscr{F}$, the length of $\mathscr{F}$ is defined as follows: $\mathscr{F}$ admits a filtration $\mathscr{F}=\mathscr{F}^{n} \supset \mathscr{F}^{n-1} \supset \cdots \supset \mathscr{F}^{0}=0$ by sheaves $\mathscr{F}^{i} / \mathscr{F}^{i-1}$ supported on residual gerbes of $\mathscr{A}$. The length of $\mathscr{F}^{i} / \mathscr{F}^{i-1}$ is defined to be its rank as a sheaf on the residual gerbe and the length of $\mathscr{F}$ is then defined to be $\ell(\mathscr{F})=\sum_{i} \ell\left(\mathscr{F}^{i} / \mathscr{F}^{i-1}\right)$.

While this definition may seem contrived, it fits naturally into a general theory of Chern classes and Hilbert polynomials for twisted sheaves. In section 2.2.7 of [15], the reader will find a proof that the length is constant in a flat family.

Definition 4.2.10. A flat family of finite length coherent $\mathscr{A}$-twisted sheaves parametrized by $S$ is an $S$-flat quasi-coherent $\mathscr{A}$-twisted sheaf $\mathscr{F}$ of finite presentation over $\mathscr{A} \times S$ such that for every geometric point $s \rightarrow S$, the fiber $\mathscr{F}_{s}$ is a coherent $\mathscr{A}_{s}$-twisted sheaf of finite length.

It is easy to see that the collection of finite length coherent $\mathscr{A}$-twisted sheaves of finite length forms an Artin stack. For details, the reader can consult [14] or section 2.3 of [15].

Definition 4.2.11. The stack of finite length coherent $\mathscr{A}$-twisted sheaves of length $\ell$ will be denoted $\mathbf{F}_{\mathscr{A} / k}^{\ell}$.

We recall a well-known result of Mukai (which carries over mutatis mutandi to the twisted case).

Proposition 4.2.12 (Mukai). For every algebraically closed extension field $K / k$, the Fourier-Mukai transform defines an equivalence of fiber categories

$$
\mathbf{F}_{\mathscr{A} / k}^{r}(K) \stackrel{\sim}{\rightarrow} \mathbf{H}_{\mathscr{T} / k}^{r}(K) .
$$

For the proof, the reader is referred to $\$ 3$ of [17]. For geometric purposes, it is useful to have the following totally unsurprising extension of the classical result. 
Proposition 4.2.13. Given any $k$-scheme $S$, the twisted Fourier-Mukai transform establishes an equivalence of categories

$$
\mathbf{F}_{\mathscr{A} / k}^{r}(S) \stackrel{\sim}{\rightarrow} \mathbf{H}_{\mathscr{T} / k}^{r}(S) .
$$

Proof. By the obvious compatibility of the Fourier-Mukai transform with derived base change, it is clear that $\Phi$ yields functors between the categories described in the proposition. Furthermore, it is clear that it suffices to prove the result assuming that $T$ has a section: any map of stacks which is an isomorphism locally on the base must be an isomorphism. (Note: here we do not mean simply stacks in groupoids, but arbitrary stacks of categories.) Arguing as in 4.1.2, the statement reduces to the obvious (twisted) relativization of Theorem 2.2 of [17].

Corollary 4.2.14. The Fourier-Mukai transform induces an isomorphism of stacks $\mathbf{F}_{\mathscr{A} / k}^{r} \stackrel{\sim}{\rightarrow} \mathbf{H}_{\mathscr{T} / k}^{r}$. In particular, $\mathbf{H}_{\mathscr{T} / k}^{r}$ is a quasi-proper Artin stack of finite presentation over the base.

Remark 4.2.15. This gives a nice example of a proof of (non-obvious) algebraicity of a very concrete stack of coherent sheaves using methods which pass through the (not as concrete) fibered category of derived categories.

Question 4.2.16. Is the stack of homogeneous coherent sheaves a locally closed substack of the stack of coherent sheaves?

4.3. A criterion for homogeneous index reduction. Let $k$ be a field, $A$ an abelian variety over $k, T$ an $A$-torsor, and $\beta \in \operatorname{Br}(k)$ a Brauer class. Choose a gerbe $\mathscr{S} \rightarrow$ Spec $k$ representing $\beta$ and let $f: \mathscr{T} \rightarrow \mathscr{S}$ denote the pullback to $T$.

Definition 4.3.1. The class $\beta$ has homogeneous index reduction if there is a complex of homogeneous $\beta$-twisted sheaves on $T$ of rank equal to $\operatorname{ind}_{k(T)}\left(\beta_{k(T)}\right)$.

Definition 4.3.2. The class $\beta$ has index reduction by degree 0 obstructions if

$$
\operatorname{ind}\left(\beta_{k(T)}\right)=\iota_{\beta}\left(\operatorname{Pic}_{T / k}^{0}, \mathscr{P} i c_{T / k}^{0}\right) .
$$

The main result of this section is the following.

Proposition 4.3.3. The class $\beta$ has homogeneous index reduction if and only if it has index reduction by degree 0 obstructions.

Proof. This follows immediately from 4.2.14, which shows that the minimal rank of a homogeneous $\mathscr{T}$-twisted sheaf equals the minimal length of an Artinian $\mathscr{A}$-twisted coherent sheaf. But the latter is computed precisely by the formula given in 4.3 .2 .

In the case of genus 1 curves, example 4.3 .4 shows that that homogeneous index reduction need not hold in general. However, it follows immediately from 2.2.3 that any genus 1 curve admits semihomogeneous index reduction.

Example 4.3.4. Let $C / k$ be a genus 1 curve with per $C \neq$ ind $C$ (for the existence of these, see Theorem 3 of [6] or [4]). By [5] Theorem 2.1.1, there is a Brauer class $\beta \in \operatorname{Br}(k)$ such that $\beta_{k(C)}=0$, however $\beta$ is not equal to any obstruction class from $\operatorname{Pic}_{E / k}^{0}$. In particular, $\beta$ does not have index reduction by degree 0 obstructions.

It follows more generally that if $T / k$ is a torsor for an abelian variety, a necessary condition that all Brauer classes have index reduction by degree 0 obstructions is that the divisorial index group and the period group of $T$ coincide (see [5], for definitions of these groups and page 5 for the relevant conclusion). On the other hand, it is not at all clear that the reverse implication should hold and that for such torsors we should have homogeneous index reduction. 


\section{APPEndix A. A PERIOD-INDEX RESUlt}

\section{BHARGAV BHATT}

Let $A \rightarrow S$ be an abelian scheme, and let $X$ be a torsor for it. Our goal is to relate the derived category of coherent sheaves on $X$ to that on a suitable $\mathbf{G}_{m}$-gerbe on $A^{t}$, the dual abelian scheme. As an application, we obtain a period-index result over global fields for geometrically trivial Brauer classes on abelian varieties coming from locally trivial torsors.

A.1. Construction of the equivalence. Let $\pi: A \rightarrow S$ be an abelian scheme, and let $\pi: A^{t} \rightarrow S$ be the dual scheme. The Leray spectral sequence for the latter (with $\mathbf{G}_{m}$-coeffecients) gives us a low degree short exact sequence

$$
1 \rightarrow \mathrm{H}^{2}\left(S, \mathbf{G}_{m}\right) \rightarrow F^{1}\left(\mathrm{H}^{2}\left(A^{t}, \mathbf{G}_{m}\right)\right) \rightarrow \mathrm{H}^{1}\left(S, \mathrm{Pic}_{A^{t} / S}\right) \rightarrow 1
$$

where $F^{\bullet}$ is the filtration defined by the spectral sequence. Note that $F^{1}\left(\mathrm{H}^{2}\left(A^{t}, \mathbf{G}_{m}\right)\right)$ can be identified as the subgroup of $\mathrm{H}^{2}\left(A^{t}, \mathbf{G}_{m}\right)$ consisting of geometrically trivial classes (i.e: classes killed by fppf localisation on $S$ ). The preceeding exact sequence is canonically split by the zero section $e^{t}: S \rightarrow A^{t}$. In particular, there's a natural (composite) map

$$
\alpha: \mathrm{H}^{1}(S, A) \cong \mathrm{H}^{1}\left(S, \operatorname{Pic}_{A^{t} / S}^{0}\right) \rightarrow \mathrm{H}^{1}\left(S, \operatorname{Pic}_{A^{t} / S}\right) \rightarrow \mathrm{H}^{2}\left(A^{t}, \mathbf{G}_{m}\right)
$$

where $A \cong \operatorname{Pic}_{A^{t} / S}^{0} \rightarrow \operatorname{Pic}_{A^{t} / S}$ is the connected component of the identity. Denote by $\alpha_{X}$ the Brauer class associated to the class $[X]$ of a torsor $X$ under this map. Then there are two ways interpret the association $X \mapsto \alpha_{X}$.

- A geometric interpretation

Given an $A$-torsor $X$, we have a canonical associated $\mathbf{G}_{m}$-gerbe $\mathbf{P i c}_{X / S} \rightarrow \mathrm{Pic}_{X / S}$. Restricting this $\mathbf{G}_{m}$-gerbe along the connected component $\mathrm{Pic}_{X / S}^{0} \rightarrow \mathrm{Pic}_{X / S}$, we obtain a $\mathbf{G}_{m}$-gerbe over $\mathrm{Pic}_{X / S}^{0}$. Since $X$ is an $A$-torsor, we have a canonical isomorphism $A^{t} \cong \operatorname{Pic}_{X / S}^{0}$. Therefore, we obtain a $\mathbf{G}_{m}$-gerbe $\mathbf{P i c}_{X / S}^{0} \rightarrow A^{t}$ whose class in $\mathrm{H}^{2}\left(A^{t}, \mathbf{G}_{m}\right)$ will be denoted by $\beta_{X}$. Note that since a torsor is geometrically trival, the functoriality of this association implies that the class we obtain lands in $F^{1}\left(\mathrm{H}^{2}\left(A^{t}, \mathbf{G}_{m}\right)\right)$.

- A homological interpretation

Given an $A$-torsor $X$, the Leray spectral sequence for $X \rightarrow S$ (with $\mathbf{G}_{m}$-coeffecients) gives us a low degree exact sequence

$$
\operatorname{Pic}(X) \rightarrow \operatorname{Pic}_{X / S}(S) \rightarrow \mathrm{H}^{2}\left(S, \mathbf{G}_{m}\right)
$$

This sequence, of course, continues to exist if we base change on $S$. Therefore, we can replace $S$ with $A^{t} \cong \mathrm{Pic}_{X / S}^{0}$, and $X$ with $X \times_{S} \operatorname{Pic}_{X / S}^{0}$ to get

$$
\operatorname{Pic}\left(X \times{ }_{S} \operatorname{Pic}_{X / S}^{0}\right) \rightarrow \operatorname{Pic}_{X / S}\left(\operatorname{Pic}_{X / S}^{0}\right) \rightarrow \mathrm{H}^{2}\left(\operatorname{Pic}_{X / S}^{0}, \mathbf{G}_{m}\right)
$$

The natural inclusion $\operatorname{Pic}_{X / S}^{0} \rightarrow \operatorname{Pic}_{X / S}$ defines a canonical element $\mathcal{P}$ (the "Poincare bundle") in $\operatorname{Pic}_{X / S}\left(\operatorname{Pic}_{X / S}^{0}\right)$. The obstruction to lifting $\mathcal{P}$ to a line bundle on $X \times_{S} \operatorname{Pic}_{X / S}^{0}$ (i.e: the failure of $\mathrm{Pic}_{X / S}^{0}$ to be a fine moduli space for translation invariant line bundles on $X$ ) defines an element $\gamma_{X}$ in $\mathrm{H}^{2}\left(\mathrm{Pic}_{X / S}^{0}, \mathbf{G}_{m}\right)=\mathrm{H}^{2}\left(A^{t}, \mathbf{G}_{m}\right)$. Once again, by functoriality of the construction, it's clear that the constructed element lies in $F^{1}\left(\mathrm{H}^{2}\left(A^{t}, \mathbf{G}_{m}\right)\right)$.

A messy cocycle calculation shows that $\alpha_{X}=-\beta_{X}=-\gamma_{X}$. Given this, we construct the desired equivalence. Specifically, we have:

Theorem A.1.1. Let $\pi: A \rightarrow S$ be an abelian scheme and let $\pi^{t}: A^{t} \rightarrow S$ be its dual scheme. Given an $A$-torsor $f: X \rightarrow S$, let $\alpha_{X} \in \mathrm{H}^{2}\left(A^{t}, \mathbf{G}_{m}\right)$ be the class defined above. Then the universal line bundle $\mathcal{P}$ on $X \times{ }_{S} \mathbf{P i c}_{X / S}^{0}$, when thought of as the kernel of a Fourier-Mukai transform, defines an equivalence of categories $\mathbf{F}: D(X) \rightarrow D\left(A^{t},-\alpha_{X}\right)$ 
Proof. This is proven in Theorem 4.1.2. We'll simply remark that the significance of the negative sign in $D\left(A^{t},-\alpha_{X}\right)$ is that the pushforward of $\mathcal{P}$ along the projection $X \times_{S} \mathbf{P i c}_{X / S}^{0} \rightarrow \mathbf{P i c}_{X / S}^{0}$ is a $\gamma_{X}$-twisted sheaf when the target is thought of as a $\mathbf{G}_{m}$-gerbe over $A^{t}$, and that $\gamma_{X}=-\alpha_{X}$.

A.2. An application. Our goal is to prove a period-index result for Brauer classes associated to torsors for abelian varieties. Specifically, we will show:

Theorem A.2.1. Let $A$ be a $g$-dimensional principally polarised abelian variety over a field $k$. Given an $A$-torsor $X$, let $\alpha_{X} \in \mathrm{H}^{2}\left(A^{t}, \mathbf{G}_{m}\right)$ be the class defined earlier. Assume one of the following

(1) $k$ has no Brauer group.

(2) $k$ is a global field and $X$ is an element of $\amalg(A)$ (i.e: has points locally).

Then ind $\left(\alpha_{X}\right) \mid \operatorname{per}(X)^{g}$. Furthermore, if $X$ has odd order in in $\mathrm{H}^{1}(k, A)$, then ind $\left(\alpha_{X}\right) \mid \operatorname{per}\left(\alpha_{X}\right)^{g}$.

A priori, one knows that with no assumptions on $k$ that ind $\left(\alpha_{X}\right)$ divides $\operatorname{per}(X)^{2 g}$ (a fact due to Lenstra, see [7]). On the other hand, in the case that $k$ is of the function field of a curve over $\mathbf{C}$, it has been conjectured (see page 12 of [8]) that one should have ind $\left(\alpha_{X}\right) \mid \operatorname{per}(X)^{g}$, based only on the fact that $A$ is a variety over $k$ of dimension $g$. By Tsen's theorem, we may apply Theorem A.2.1 and verify that the conjecture holds in this case.

The idea of the proof is to use Theorem A.1.1 to construct an $\alpha_{X}$-twisted vector bundle of rank $\operatorname{per}(X)^{g}$ on $A^{t}$. In order to do so, however, we need a good supply of sheaves on $X$.

Lemma A.2.2. Assume we're in the situation of Theorem A.2.1. If $L \in \operatorname{Pic}(A)$ is a line bundle defining a principal polarisation, then the class of $L^{\mathrm{per}(X)}$ in $\mathrm{NS}_{A} \cong \mathrm{NS}_{X}$ is effective (representable by a line bundle on $X$ ).

Proof. We'll think of the torsor $X$ as an extension

$$
1 \rightarrow A \rightarrow T \rightarrow \mathbf{Z} \rightarrow 1
$$

where the fibre of $T \rightarrow \mathbf{Z}$ over $1 \in \mathbf{Z}$ is the torsor $X$. Given a line bundle $L \in \operatorname{Pic}(A)$ which defines a principal polarisation $\varphi_{L}: A \rightarrow A^{t}$, we can push this exact sequence out along $\varphi_{L}$ to obtain an extension

$$
1 \rightarrow A^{t} \rightarrow T^{\prime} \rightarrow \mathbf{Z} \rightarrow 1
$$

which corresponds to the image of $X$ under $\mathrm{H}^{1}\left(\varphi_{L}\right): \mathrm{H}^{1}(k, A) \rightarrow \mathrm{H}^{1}\left(k, A^{t}\right)$. As a class in $\operatorname{Ext}_{k}^{1}\left(\mathbf{Z}, A^{t}\right)$, this has order $\operatorname{per}(X)$. On the other hand, since $X$ is an $A$-torsor, we have canonical isomorphisms $A^{t} \cong \mathrm{Pic}_{X}^{0}$ and $\mathrm{NS}_{A} \cong \mathrm{NS}_{X}$. Thus, we obtain a canonical extension

$$
1 \rightarrow A^{t} \rightarrow \mathrm{Pic}_{X} \rightarrow \mathrm{NS}_{A} \rightarrow 1
$$

The class of the line bundle $L$ in $\mathrm{NS}_{A}$ defines a morphism $\mathbf{Z} \rightarrow \mathrm{NS}_{A}$. Pulling the preceeding exact sequence back along this morphism, we obtain an extension

$$
1 \rightarrow A^{t} \rightarrow T^{\prime \prime} \rightarrow \mathbf{Z} \rightarrow 1
$$

A messy calculation with cocyles reveals that the class of this extension in $\operatorname{Ext}_{k}^{1}\left(\mathbf{Z}, A^{t}\right)$ is the inverse of the class associated to extension (2). The upshot of this is that extension (3) also has order per $(X)$ in the group of extension classes. This means that there is an element $M \in \operatorname{Pic}_{X}(k)$ whose class $\mathrm{NS}_{X} \cong \mathrm{NS}_{A}$ is the same as that of $L^{\operatorname{per}(X)}$. What remains to be shown now is that $M \in \operatorname{Pic}_{X}(k)$ comes from an actual line bundle on $X$. The obstruction to $M$ being represented by a line bundle lives in $\operatorname{Br}(k)$. In the case that $\operatorname{Br}(k)=0$ we are obviously done. In the case of global fields, recall that an element of $\operatorname{Br}(k)$ is trivial if it is locally trivial. By functoriality of the obstruction and the fact that $X$ is locally split, we see that there is no obstruction locally. Thus, there is no global obstruction to representing $M$ by an actual line bundle in this case as well.

We're now in a position to give a proof of Theorem A.2.1

Proof of Theorem A.2.1 Note that the kernel of

$$
\alpha: \mathrm{H}^{1}(k, A) \rightarrow \mathrm{H}^{1}\left(k, \mathrm{Pic}_{A^{t}}\right) \rightarrow \mathrm{H}^{2}\left(A^{t}, \mathbf{G}_{m}\right)
$$


is 2-torsion because the kernel of the first map is 2-torsion 1 and the second map is injective. Thus, if $X$ has odd order in $\mathrm{H}^{1}(k, A)$, then its image $\alpha_{X}$ under the map $\alpha$ has the same order. Thus, it suffices to show that ind $\left(\alpha_{X}\right) \mid \operatorname{per}(X)^{g}$.

Employing the notation of LemmaA.2.2, we know that there exists a line bundle $M$ on $X$ whose class in $\mathrm{NS}_{X / S} \cong \mathrm{NS}_{A / S}$ is the class of $L^{\mathrm{per}(X)}$. In this situation, using the notation of Theorem A.1.1, I claim that $\mathbf{F}(M)$ is an $\alpha_{X}$-twisted vector bundle, and that it has rank per $(X)^{g}$. Both these assertions are fppf local on the base and, therefore, we may assume that $k$ is algebraically closed and, consequently, that $X$ is trivial and $\mathbf{F}$ is the regular Fourier-Mukai functor, upto tensoring by line bundles on $A^{t}$ (which is the same as changing the choice of trivialisation of $X$ ). Since the property of being a vector bundle of a specified rank is invariant under tensoring by line bundles, we may assume that $\mathbf{F}$ is the regular Fourier-Mukai functor. Thus, we're reduced to proving that if $M$ is line bundle on $A$ whose class in $\mathrm{NS}_{A / S}$ is the same as that of $L^{n}$, then $\mathbf{F}(M)$ is a vector bundle, and has rank $n^{g}$.

Since $M$ is non-degenerate, $\mathbf{F}(M)$ is a vector bundle (upto a translation in the derived category). To check that it has the right rank, recall the Fourier-Mukai functor turns Euler characteristics into ranks, upto a sign. Thus, we need to show $\chi(M)= \pm n^{g}$. We know that $\chi(M)^{2}=\# K(M)$, where $K(M)$ is the kernel of the isogeny $\varphi_{M}: A \rightarrow A^{t}$. Since $\varphi_{M}=\varphi_{L^{n}}$, we have $\chi(M)^{2}=\# K\left(L^{n}\right)$. Since $L$ is a principal polarisation, $K(L)$ is trivial. Therefore, $\# K\left(L^{n}\right)=A[n]$ which has rank $n^{2 g}$. Thus, $\chi(M)= \pm n^{g}$, as was required.

\section{REFERENCES}

[1] M. Artin. Versal deformations and algebraic stacks. Invent. Math., 27:165-189, 1974.

[2] M. F. Atiyah. Vector bundles over an elliptic curve. Proc. London Math. Soc. (3), 7:414-452, 1957.

[3] Siegfried Bosch, Werner Lütkebohmert, and Michel Raynaud. Néron models, volume 21 of Ergebnisse der Mathematik und ihrer Grenzgebiete (3) [Results in Mathematics and Related Areas (3)]. Springer-Verlag, Berlin, 1990.

[4] J. W. S. Cassels. Arithmetic on curves of genus 1. V. Two counterexamples. J. London Math. Soc., 38:244-248, 1963.

[5] Mirela Ciperiani and Daniel Krashen. Relative Brauer groups of genus 1 curves. arXiv:math.NT/0701614

[6] Pete L. Clark. The period-index problem in WC-groups. I. Elliptic curves. J. Number Theory, 114(1):193-208, 2005.

[7] Peter L. Clark. Period-index problems in WC-groups II: abelian varieties. preprint available : http://www.math.uga.edu/ pete/papers.html.

[8] Jean-Louis Colliot-Thélène. Die Brauersche Gruppe; ihre Verallgemeinerungen und Anwendungen in der Arithmetischen Geometrie, 2001. Unpublished lecture notes, available at http://www.math.u-psud.fr/ colliot/liste-cours-exposes.html.

[9] A. J. de Jong. The period-index problem for the Brauer group of an algebraic surface. Duke Math. J., 123(1):71-94, 2004.

[10] Jean Giraud. Cohomologie non abélienne. Springer-Verlag, Berlin, 1971. Die Grundlehren der mathematischen Wissenschaften, Band 179.

[11] Daniel Huybrechts and Manfred Lehn. The geometry of moduli spaces of sheaves. Aspects of Mathematics, E31. Friedr. Vieweg \& Sohn, Braunschweig, 1997.

[12] Gérard Laumon and Laurent Moret-Bailly. Champs algébriques, volume 39 of Ergebnisse der Mathematik und ihrer Grenzgebiete. 3. Folge. A Series of Modern Surveys in Mathematics [Results in Mathematics and Related Areas. 3rd Series. A Series of Modern Surveys in Mathematics]. Springer-Verlag, Berlin, 2000.

[13] Max Lieblich. Twisted sheaves and the period-index problem, 2004. preprint.

[14] Max Lieblich. Remarks on the stack of coherent algebras. Int. Math. Res. Not., pages Art. ID 75273, $12,2006$.

[15] Max Lieblich. Moduli of twisted sheaves. Duke Math. J., 138(1):23-118, 2007.

[16] A. S. Merkurjev, I. A. Panin, and A. R. Wadsworth. Index reduction formulas for twisted flag varieties. I. K-Theory, 10(6):517-596, 1996.

[17] Shigeru Mukai. Duality between $D(X)$ and $D(\hat{X})$ with its application to Picard sheaves. Nagoya Math. J., 81:153-175, 1981.

[18] D. O. Orlov. Derived categories of coherent sheaves on abelian varieties and equivalences between them. Izv. Ross. Akad. Nauk Ser. Mat., 66(3):131-158, 2002.

[19] I. A. Panin. On the algebraic $K$-theory of twisted flag varieties. $K$-Theory, 8(6):541-585, 1994.

[20] Bjorn Poonen and Michael Stoll. The Cassels-Tate pairing on polarized abelian varieties. Ann. of Math. (2), 150(3):11091149, 1999.

[21] Peter Roquette. Splitting of algebras by function fields of one variable. Nagoya Math. J., 27:625-642, 1966.

\footnotetext{
${ }^{1}$ There is a short exact sequence $\mathrm{H}^{0}\left(k, \mathrm{NS}_{A^{t}}\right) \rightarrow \mathrm{H}^{1}(k, A) \rightarrow \mathrm{H}^{1}\left(k, \mathrm{Pic}_{A^{t}}\right)$. The automorphism -1 of $A^{t}$ acts by 1 on $\mathrm{H}^{0}\left(k, N S_{A^{t}}\right)$ and by -1 on $\mathrm{H}^{1}(k, A)$. Thus the image of the first map, which is the kernel of the second map, is 2 -torsion. This image can, however, be non-zero - this phenomenon is explored in much greater depth in [20] where, amongst other things, the non-triviliality of this image is identified to be the reason (over a global field $k$ ) the order of $\amalg(A)$ can be a non-square, assuming it is finite.
} 
[22] Aidan Schofield and Michel Van den Bergh. The index of a Brauer class on a Brauer-Severi variety. Trans. Amer. Math. Soc., 333(2):729-739, 1992.

[23] Angelo Vistoli. Intersection theory on algebraic stacks and on their moduli spaces. Invent. Math., 97(3):613-670, 1989.

[24] V. I. Yanchevski1. Index reduction formulas for local hyperelliptic curves with poor reduction. Dokl. Akad. Nauk Belarusi, 41(1):16-21, 122, 1997.

Yale University, New Haven, CT

E-mail address: daniel.krashen@yale.edu

Princeton University, PRINCETON, NJ

E-mail address: lieblich@math.princeton.edu

Princeton University, Princeton, NJ

E-mail address: bbhatt@math.princeton.edu 